\title{
HARMONIC MORPHISMS AND CONFORMAL FOLIATIONS BY GEODESICS OF THREE-DIMENSIONAL SPACE FORMS
}

\author{
PAUL BAIRD and JOHN C. WOOD
}

(Received 15 October 1989)

Communicated by J. H. Rubinstein

\begin{abstract}
A complete classification is given of harmonic morphisms to a surface and conformal foliations by geodesics, with or without isolated singularities, of a simply-connected space form. The method is to associate to any such a holomorphic map from a Riemann surface into the space of geodesics of the space form. Properties such as nonintersecting fibres (or leaves) are translated into conditions on the holomorphic mapping which show it must have a simple form corresponding to a standard example.
\end{abstract}

1980 Mathematics subject classification (Amer. Math. Soc.) (1985 Revision): 53 C 12, 58 E 20, 31 B 99.

\section{Introduction}

A harmonic morphism is a smooth map $\phi: M \rightarrow N$ between Riemannian manifolds such that if $f$ is a real-valued harmonic function on an open set $V$ of $N$ and $\phi^{-1}(V) \subset M$ is nonempty, the composition $f \circ \phi$ is harmonic on $\phi^{-1}(V)$. Harmonic morphisms may be characterized as harmonic maps which are horizontally weakly conformal $[9,16]$, or as Brownian path preserving mappings [5]. Examples are (i) orthogonal projection $\mathbb{R}^{3} \rightarrow \mathbb{R}^{2}$, and (ii) the Hopf map $S^{3} \rightarrow S^{2}$.

In [4] we showed that these are essentially the only nonconstant harmonic morphisms from $\mathbf{R}^{3}$ or $S^{3}$ to a surface. In the present paper we complete our treatment of harmonic morphisms from simply-connected three-dimensional

(C) 1991 Australian Mathematical Society 0263-6115/91 \$A2.00+0.00 
space forms by considering harmonic morphisms from hyperbolic 3-space $\mathbf{H}^{3}$ to a surface. We shall show that there are essentially two non-constant harmonic morphisms: (iii) orthogonal projection $\mathbb{H}^{3} \rightarrow \mathbb{H}^{2}$; and (iv) projection $\mathbb{H}^{3} \rightarrow \mathbb{C}$ to the plane at infinity; see Examples 1.6 for definitions. More precisely, we have

THeOREM 3.5. Any nonconstant harmonic morphism from $\mathbb{H}^{3}$ to a Riemannian 2-manifold $N$ is, up to isometry of $\mathbb{H}^{3}$, orthogonal projection $\mathbb{H}^{3} \rightarrow$ $\mathbb{H}^{2}$ or projection $\mathbb{H}^{3} \rightarrow \mathbb{C}$ to the plane at infinity followed by a weakly conformal map of $\mathbb{H}^{2}$ or $\mathbb{C}$ to $N$.

Together with [4, Theorems 4.1 and 5.1] and facts about harmonic morphisms into codomains of other dimensions, this completely describes all harmonic morphisms from a simply-connected three-dimensional space form.

A conformal foliation of a Riemannian manifold is one given locally by conformal submersions (see [20] and Section 1C), a Riemannian foliation is a special case. Given a submersive harmonic morphism $\phi: M \rightarrow N$, the fibres $\phi^{-1}(y), y \in N$, define a conformal foliation, and further if $N$ is two-dimensional, the fibres are minimal [3]. In particular, to a submersive harmonic morphism from a three-dimensional manifold $M$ to a surface $N$ is associated a conformal foliation of $M$ by geodesics. There is an inverse construction; see Section $2 \mathrm{C}$. Applying this construction to examples (i) to (iv) above gives four standard foliations. Theorem 3.5 follows from its counterpart for foliations: up to isometry, the only conformal foliations by geodesics of a simply-connected space form $\mathbb{R}^{3}, S^{3}$ or $\mathbb{H}^{3}$ are the four standard foliations (see Theorems 2.14, 2.15 and 3.3).

For harmonic morphisms which are not submersions we need a factorization theorem (Theorem 2.19) which shows that any harmonic morphism from a convex domain $U$ of a three-dimensional space form to a surface can be factored into a submersive harmonic morphism followed by a weakly conformal map. This allows us to associate a conformal foliation by geodesics to an arbitrary nonconstant harmonic morphism of $U$ to a surface.

In $\S 4$ we study harmonic morphisms from space forms with isolated sinzularities. We first show (Theorem 4.2 ) that the only possible singularity is radial projection, typified by the harmonic morphism $\phi: \mathbb{R}^{3} \backslash\{0\} \rightarrow S^{2}$, $x \mapsto x /|x|$. We then give global implications showing that any non-constant harmonic morphism with isolated singularities from $\mathbb{R}^{3}, S^{3}$ or $\mathbb{H}^{3}$ to a surface is, up to isometry, a standard example (a radial projection) followed by a weakly conformal map (Theorem 4.2).

Again these results follow from a related result on conformal foliations by jeodesics with singularities (Theorem 4.1) 
The main idea of our proofs is that the locations of the leaves of a foliation by geodesics of a convex open subset $U$ of a space form are described by a map $i$ of the leaf space into the space $G_{U}$ of geodesics of $U$. This mapping is holomorphic if and only if the foliation is conformal. This gives a unified treatment of the local theory of harmonic morphisms from a threedimensional space form to a surface. We prove our main results by studying the global behaviour of the holomorphic mapping $i$. Constant curvature is used in an essential way, for example only in this case do we know that the space of geodesics is a complex surface (cf. [15]). Furthermore our factorization theorem (Theorem 2.19) relies on an extension of certain functions which are harmonic in the presence of constant curvature.

Let $M$ be a non-simply-connected three-dimensional space form. Then $M=\mathbb{E}^{3} / \Gamma$ where $\mathbb{E}^{3}=\mathbb{R}^{3}, S^{3}$ or $\mathbb{H}^{3}$ and $\Gamma$ is a discrete group of isometries acting freely on $\mathbb{E}^{3}$. Any harmonic morphism or conformal foliation by geodesics of $M$ lifts to one of $\mathbb{E}^{3}$. Thus our results imply results on harmonic morphisms and conformal foliations by geodesics of non-simplyconnected three-dimensional space forms. As an example we give a complete classification of harmonic morphisms to a surface and conformal foliations by geodesics, with or without isolated singularities, of $\mathbb{R} P^{3}$ in Section 5 showing that any such is, up to isometry, a standard example obtained from standard examples on $S^{3}$.

\section{Preliminaries}

(A) Space forms [21]. Let $\mathbb{E}^{m}$ denote a simply-connected space form, that is, a simply-connected complete Riemannian manifold of constant sectional curvature $K$. By scaling we may assume $K=+1,0$ or -1 . Then $\mathbb{E}^{m}$ is isometric to a sphere $S^{m}$ if $K=+1$, Euclidean space $\mathbb{R}^{m}$ if $K=0$, or hyperbolic space $\mathbb{H}^{m}$ if $K=-1$. Note all these manifolds have standard orientations. We describe three models for $\mathbb{H}^{m}$ that we shall use.

(i) The Poincare model. Here $\mathbb{H}^{m}=$ the open unit ball $D^{m}=\left\{x \in \mathbb{R}^{m}\right.$ : $|x|<1\}$ with the metric

$$
d s^{2}=\frac{4 \sum_{i=1}^{m} d x_{i}^{2}}{\left(1-|x|^{2}\right)^{2}} .
$$

$\left(|\cdot|\right.$ will always denote the Euclidean norm $\left.|x|=\sqrt{ }\left(x_{i}^{2}+\cdots+x_{m}^{2}\right).\right)$ The maximal geodesics of $\mathbb{H}^{m}$ are the intersections of Euclidean circles with $D^{m}$ which meet the boundary $\partial D^{m}$ at right angles.

(ii) The Beltrami model. Again $\mathbb{H}^{m}=$ the open unit ball. The maximal geodesics of $\mathbb{H}^{m}$ are the intersections of Euclidean lines with $D^{m}$. The 


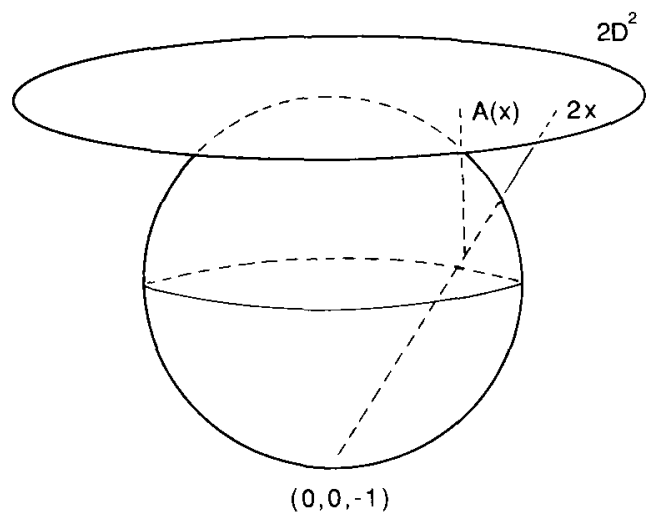

FIGURE 1

Poincare and Beltrami models are related by the diffeomorphism $A: D^{m} \rightarrow$ $D^{m}$

$$
A(x)=\frac{2 x}{1+|x|^{2}},
$$

(homeomorphism on $\overline{D^{m}}$ ) which sends any circle orthogonal to $\partial D^{m}$ (that is, a geodesic of the Poincare model) to the straight line joining the same points of $\partial D^{m}$ (that is, a geodesic of the Beltrami model). (This can be seen by noting that $A$ is the composition

$$
D^{m} \stackrel{2}{\rightarrow} 2 D^{m} \stackrel{\sigma^{-1}}{\rightarrow} H_{+}^{m} \stackrel{v}{\rightarrow} D^{m}
$$

where the first map sends $x$ to $2 x, \sigma: H_{+}^{m} \rightarrow 2 D^{m}$ is stereographic projection through $(0, \ldots, 0,-1)$ of the open upper hemisphere $H_{+}^{m}$ of the unit sphere $S^{m}$ to the tangent plane at $(0, \ldots, 0,1)$ and $v$ is vertical projection $\left(x_{1}, \ldots, x_{m+1}\right) \rightarrow\left(x_{1}, \ldots, x_{m}\right)$.) (See Figure 1.)

(iii) The half-space model. Here $\mathbb{H}^{m}$ is the upper half-space

$$
\mathbb{R}_{+}^{m}=\left\{\left(x_{1}, \ldots, x_{m}\right) \in \mathbb{R}^{m}: x_{m}>0\right\}
$$

with the metric

$$
d s^{2}=\frac{\sum_{i=1}^{m} d x_{i}^{2}}{x_{m}^{2}} .
$$

This is related to the Poincare model by an isometry called the Cayley transformation; see [21, page 71].

(B) Harmonic morphisms. Let $\phi: M \rightarrow N$ be a smooth mapping between Riemannian manifolds $M=M^{m}, N=N^{n}$ of dimensions $m$ and $n$ respectively. All Riemannian manifolds will be assumed connected and smooth (that is, $C^{\infty}$ ) unless otherwise stated. For each $x \in M$, let $V_{x}=\operatorname{ker} d \phi_{x}$ denote the vertical space at $x$, and let $H_{x}$ denote its orthogonal complement 
in $T_{x} M$ called the horizontal space at $x$. Thus $T_{x} M$ decomposes as the direct sum $T_{x} M=H_{x} \oplus V_{x}$. We denote by $V$ and $H$ the corresponding vertical and horizontal distributions in the tangent bundle $T M$. Then $\phi$ is said to be horizontally (weakly) conformal if, for each $x \in M$ where $d \phi_{x} \neq 0$, the restriction of $d \phi_{x}$ to $H_{x}$ is conformal and surjective onto $T_{\phi(x)} N$. Thus there is some number $\lambda(x) \in(0, \infty)$, called the conformality factor or dilation of $\phi$ at $x$, such that $\left|d \phi_{x}(X)\right|=\lambda(x)|X|$ for each $X \in H_{x}$. Setting $\lambda$ equal to zero at critical points (that is, points $x \in M$ where $d \phi_{x}=0$ ), we obtain a continuous function $\lambda: M \rightarrow \mathbb{R}$ called the dilation of $\phi$. Note that $\lambda^{2}=|d \phi|^{2} / n$ is smooth. The set of critical points is called the critical set of $\phi$.

We refer the reader to [4] for the basic properties of and further references to harmonic morphisms.

Harmonic morphisms to surfaces, that is, two-dimensional Riemannian manifolds, have a particularly nice characterization established in the case that $M$ is an open subset of $\mathbb{R}^{m}$ in [5] and in the general case in [3].

THeOREM 1.1 [3]. Let $\phi: M^{m} \rightarrow N^{2}$ be a submersion. Then $\phi$ is a harmonic morphism if and only if it is horizontally conformal and its fibres $\phi^{-1}(y)$ $\left(y \in N^{2}\right)$ are minimal in $M^{m}$.

Using this criterion it is easy to see that the following are harmonic morphisms:

EXAMPLes 1.2. (i) Orthogonal projection $\phi: \mathbb{R}^{3} \rightarrow \mathbb{R}^{2}=\mathbb{C}, \phi\left(x_{1}, x_{2}, x_{3}\right)$ $=\left(x_{1}, x_{2}\right)=x_{1}+i x_{2}$.

(ii) The Hopf map $\phi: S^{3} \rightarrow S^{2}$.

(iii) Orthogonal projection $\phi: \mathbb{H}^{3} \rightarrow \mathbb{H}^{2}$. This is defined as follows. Represent $\mathbb{H}^{3}$ by the Poincaré model. Then the central disc $D^{2}=\left\{\left(x_{1}, x_{2}, 0\right) \in\right.$ $\left.\mathbb{R}^{3}: x_{1}^{2}+x_{2}^{2}<1\right\}$ may be identified with $\mathbb{H}^{2}$, again by the Poincaré model. For $x \in \mathbb{H}^{3}=D^{3}$, let $\phi(x)$ be the intersection with $\mathbb{H}^{2}=D^{2}$ of the unique hyperbolic geodesic through $x$ which meets $\mathbb{H}^{2}$ orthogonally. The fibres of $\phi$ are shown in Figure 2(a). Horizontal conformality at points $\left(0,0, x_{3}\right)$ is a consequence of rotational symmetry about the $x_{3}$-axis. Horizontal conformality at other points follows by applying a suitable isometry. In fact the group of isometries of $\mathbb{H}^{3}$ induced by the Möbius transformations $z \mapsto(z-a) /(z-1 / \bar{a})$ on the boundary preserves the foliation and acts transitively on the leaves.

By elementary geometry one can construct the formula for $\phi$ which is 


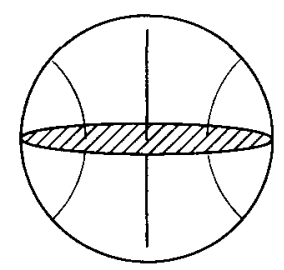

(a) Fibres of orthogonal projection $\mathbf{H}^{3} \rightarrow \mathbf{H}^{2}$

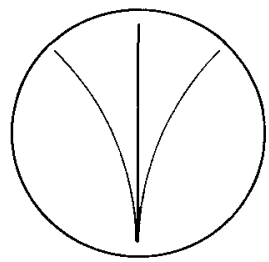

(b) Fibres of projection to the plane at infinity

FIGURE 2. Standard harmonic morphisms and corresponding conformal foliations of $\mathbf{H}^{3}$.

given by

$$
\phi\left(x_{1}, x_{2}, x_{3}\right)=\frac{1+|x|^{2}-\left\{1-2\left(x_{1}^{2}+x_{2}^{2}-x_{3}^{2}\right)+|x|^{4}\right\}^{1 / 2}}{2\left(x_{1}^{2}+x_{2}^{2}\right)}\left(x_{1}, x_{2}\right) .
$$

(iv) Projection to the plane at infinity $\phi: \mathbb{H}^{3} \rightarrow \mathbb{C}$. To define this, represent $\mathbb{H}^{3}$ by the upper half-space $\mathbb{R}_{+}^{3}=\left\{\left(x_{1}, x_{2}, x_{3}\right): x_{3}>0\right\}$ and define $\phi: \mathbb{H}^{3} \rightarrow$ $\mathbb{R}^{2}=\mathbb{C}$ by

$$
\phi\left(x_{1}, x_{2}, x_{3}\right)=\left(x_{1}, x_{2}\right)=x_{1}+i x_{2} .
$$

The fibres of $\phi$ are vertical half-lines. If we use the Poincare model $\phi$ can be described as follows. Let $x_{0}=(-1,0,0) \in \partial D^{3}$. Identify $\partial D^{3} \backslash\left\{x_{0}\right\}=S^{2} \backslash\left\{x_{0}\right\}$ with $\mathbb{C}$ by stereographic projection from $x_{0}$. Then $\phi(x)=$ intersection of the hyperbolic geodesic through $x$ and $x_{0}$ with $\partial D^{3} \backslash\left\{x_{0}\right\}$. The fibres of $\phi$ are the geodesics emanating from $x_{0}$ (see Figure 2(b)).

Corollary 3.7 states that these four examples are essentially the only nonconstant harmonic morphisms from a simply-connected three-dimensional space form to a two-dimensional Riemannian manifold.

A further class of harmonic morphisms is provided by radial projections. Let $B^{3}$ be a geodesic ball centre $p_{0}$ in a three-dimensional space form. Let $x=\left(x_{1}, x_{2}, x_{3}\right)$ denote normal coordinates centred on $p_{0}$ (that is, coordinates given by the exponential map at $p_{0}$ ). Then by radial projection to $p_{0}$ we mean the map $\phi: B^{3} \backslash\left\{p_{0}\right\} \rightarrow S^{2}$ given by

$$
\phi(x)=\frac{x}{|x|} \text {. }
$$

The fibres of $\phi$ are the "radial" geodesics of $B^{3}$ emanating from $p_{0}$. Radial projection extends to the following harmonic morphisms which we continue to call radial projections.

EXAMPLES 1.3. (i) $\phi: \mathbb{R}^{3} \backslash\{0\} \rightarrow S^{2}$ defined by $\phi(x)=x /|x|$ (radial projection to 0 ). 
(ii) $\phi: S^{3} \backslash\{(1,0,0,0),(-1,0,0,0)\} \rightarrow S^{2}$ defined by $\phi(\cos t, \sin t x)$ $=x, t \in(0, \pi), x \in S^{2}$. The fibres of $\phi$ are half great circles through the "poles" $(1,0,0,0),(-1,0,0,0):$ this map extends radial projection to either of those points and will be called radial projection to $( \pm 1,0,0,0)$.

(iii) With the Poincaré model for $\mathbb{H}^{3}, \phi: \mathbb{H}^{3} \backslash\{0\}=D^{3} \backslash\{0\} \rightarrow S^{2}$ defined by $\phi(x)=x /|x|$ (radial projection to 0 ).

These may be considered as harmonic morphisms with isolated singularities of $\mathbb{R}^{3}, S^{3}, \mathbb{H}^{3}$. We prove in Section 4 that the only possible isolated singularity of a harmonic morphism from a three-dimensional space form to a two-dimensional Riemannian manifold is radial projection (Theorem 4.1) and that any harmonic morphism with an isolated singularity from a domain of a simply-connected space form must be, up to isometry, the restriction of one of Examples 1.3.

(C) Conformal foliations [20]. We give here a treatment of conformal foliations adapted to our purposes, there being no suitable reference.

Let $M^{m}$ be a smooth Riemannian manifold. A smooth distribution $V$ of dimension $k$, codimension $q=m-k$, is a smooth subbundle of rank $k$ of the tangent bundle. A smooth distribution which is integrable is called a $C^{\infty}$ foliation and the connected components of the integrable submanifolds of $V$ are called the leaves of the foliation. We denote by $H$ the orthogonal distribution; we shall often call $V$ (respectively $H$ ) the vertical (respectively horizontal) distribution. Given $x_{0} \in M^{m}$ there is a neighbourhood $U$ of $x_{0}$ and a diffeomorphism $\psi: U \rightarrow U_{1} \times U_{2} \subset \mathbb{R}^{k} \times \mathbb{R}^{q}$ such that the intersection of any leaf with $U$ is given by $\psi^{-1}\left(U_{1} \times y\right)$ for some $y \in U_{2}$. Such a $\psi$ is called a foliated chart and the corresponding submersion $\pi_{2} \circ \psi: U \rightarrow$ $U_{2} \subset \mathbb{R}^{q}$, where $\pi_{2}: \mathbb{R}^{k} \times \mathbb{R}^{q} \rightarrow \mathbb{R}^{q}$ is projection onto the second factor, is called a distinguished submersion. More generally, for $0 \leq r \leq \infty$, or $r=\omega$ (real analytic), $C^{r}$ foliations on a $C^{r}$ manifold can be defined in terms of foliated charts or distinguished submersions; see, for example, [12]. Note that in particular given a $C^{r}$ submersion $\phi: M^{m} \rightarrow N^{q}$, the components of the fibres (inverse images) of $\phi$ form the leaves of a $C^{r}$ foliation called the foliation associated to the submersion $\phi$.

We next define conformal foliation. Let $\stackrel{0}{\nabla}$ be the Bott partial connection on the horizontal bundle $H$ defined by

$$
\stackrel{0}{\nabla}_{W} X=\mathscr{H}\left(\mathscr{L}_{W} X\right)
$$

for $W \in C^{\infty}(V), X \in C^{\infty}(H)$. Here $\mathscr{H}$ denotes orthogonal projection onto $H$ and $\mathscr{L}_{W} X=[W, X]$ denotes the Lie derivative (or Lie bracket). Let $g_{H}$ denote the metric on $M^{m}$ restricted to $H$, that is, $g_{H}(Y, Z)=$ $g(\mathscr{H} Y, \mathscr{H} Z)$, where $g$ is the metric tensor on $M^{m}$. Let $\stackrel{0}{\nabla}$ now denote 
the induced connection on $\odot^{2} H^{*}$, the bundle of symmetric bilinear forms on $H$.

Definition 1.4. A foliation is conformal if, at all points $x \in M^{m}$,

$$
\stackrel{0}{\nabla}_{W} g_{H}=\lambda(W) g_{H}
$$

for all $W \in V_{x}$ where $\lambda(W)$ is a positive number depending only on $W$. As a special case if $\lambda(W) \equiv 0$ for all $W \in V$, the foliation is called Riemannian [18].

This definition can be understood more explicitly in several ways. Call a vector field $X$ basic (or foliate or projectable) if $\mathscr{L}_{W} X \in C^{\infty}(V)$ for all $W \in C^{\infty}(V)$. This can be interpreted [18, Lemma 4.5] as saying that $X$ is an infinitesimal automorphism of the foliation. Equivalently, for any distinguished submersion $\pi: U \rightarrow U_{2} \subset \mathbb{R}^{q}, X$ projects to a vector field on $U_{2}$. A vector field $X$ is basic if and only if its horizontal part $\mathscr{E} X$ is basic. The condition for this may be written $\stackrel{0}{\nabla}_{W}(\mathscr{H} X)=0$, that is, $a$ horizontal basic vector field is simply a horizontal vector field which is parallel with respect to the Bott partial connection. (Note that the concept of a vector field along a leaf being basic is well-defined.)

Now we restrict to the case of a foliation with trivial linear holonomy. Since the linear holonomy along a leaf coincides with that arising from the Bott partial connection [17], the holonomy of the latter is also trivial. Thus if $x$ and $y$ lie on the same leaf, parallel transport along the leaf gives a welldefined isomorphism $\tau_{x y}: H_{x} \rightarrow H_{y}$. Indeed if $X_{x} \in H_{x}$, let $X$ be the unique basic vector field along the leaf with that value at $x$; then $\tau_{x y}\left(X_{x}\right)=$ $X_{y}$, the value of $X$ at $y$.

Proposition 1.5. A foliation is conformal (respectively Riemannian) if and only if parallel transport of horizontal vector fields along the leaves is conformal (respectively isometric).

Proof. For any horizontal vector fields $X, Y$,

$$
\left(\stackrel{0}{\nabla}_{W} g_{H}\right)(X, Y)=W\left\{g_{H}(X, Y)\right\}-g_{H}\left(\stackrel{0}{\nabla}_{W} X, Y\right)-g_{H}\left(X, \stackrel{0}{\nabla}_{W} Y\right) .
$$

Now if $X$ and $Y$ are basic, the last two terms vanish. Thus from Definition 1.8 , the foliation is conformal if and only if for all basic horizontal vector fields $X, Y$,

$$
W\left\{g_{H}(X, Y)\right\}=\lambda(W) g_{H}(X, Y),
$$

which is clearly equivalent to parallel transport being conformal. 
Proposition 1.5 can be phrased as follows: a foliation is conformal (respectively Riemannian) if and only if its linear holonomy pseudogroup is a subgroup of the group of conformal (respectively isometric) transformations.

Next we have the following useful characterization in terms of distinguished submersions:

Proposition 1.6 (cf. [20]). A foliation $\mathscr{F}$ is conformal if and only if, for each distinguished submersion $\pi: U \rightarrow U_{2} \subset \mathbb{R}^{q}, U_{2}$ can be given $a$ Riemannian metric (or a conformal equivalence class of such) such that $\pi$ is a horizontally conformal submersion.

Proof. Since a basic vector field is precisely one which projects to a vector field on $U_{2}$ for any distinguished submersion, we see that parallel transport commutes with the differential of $\pi$. Thus, if $\mathscr{F}$ is conformal, if we choose a Riemannian metric on $\pi(U)$ such that $\pi$ is horizontally conformal at one point of a fibre, it will be horizontally conformal at all points.

Conversely, if $\pi$ is horizontally conformal at all points of a fibre, parallel transport is conformal and so by Proposition 1.5 the foliation is conformal.

REMARK 1.7. To check that a foliation is conformal it suffices to check that for each point $x$ of $M^{m}$, there is a distinguished submersion on a neighbourhood of $x$ with the property in the last proposition. In particular the foliation associated to a conformal submersion is conformal.

We now specialize to the case of a codimension two foliation $\mathscr{F}$, still with trivial linear holonomy, which is transversally orientable [12], that is, each horizontal space $H_{x}$ can be given an orientation in a consistent manner. Then, for each $x$, we have an "almost complex structure" $J=J_{x}: H_{x} \rightarrow H_{x}$, that is, an isometry with $J_{x}^{2}=-$ identity. We then have characterizations of conformality of $\mathscr{F}$ in terms of $J$.

Proposition 1.8. The following conditions on $\mathscr{F}$ are equivalent:

(i) $\mathscr{F}$ is conformal;

(ii) $\stackrel{0}{\nabla}_{W} J=0$ for all $W \in V$, where $\stackrel{0}{\nabla}$ is the (partial) connection induced on the bundle of linear maps $L(H, H)$ from the Bott partial connection on $H$;

(iii) parallel transport commutes with $J$;

(iv) $J$ maps horizontal basic vector fields to horizontal basic vector fields.

Proof. Since parallel transport preserves orientation it is conformal if and only if it commutes with $J$, showing equivalence of (i) and (iii). Now (iii) 
is equivalent to $\stackrel{0}{\nabla}_{W}(J X)=J\left(\stackrel{0}{\nabla}_{W} X\right)$ for all horizontal vector fields $X$ and $W \in V$. Since

$$
\left(\stackrel{0}{\nabla}_{W} J\right)(X)=\stackrel{0}{\nabla}_{W}(J X)-J\left(\stackrel{0}{\nabla}_{W} X\right),
$$

this is equivalent to (ii). Lastly, (ii) holds if and only if $\stackrel{0}{\nabla}_{W}(J X)=J\left(\stackrel{0}{\nabla}_{W} X\right)$ for all horizontal basic vector fields $X$, that is, if and only if $J X$ is basic for all horizontal basic vector fields $X$, which is (iv).

\section{Local theory of harmonic morphisms on a three-dimensional space form}

(A) Space of geodesics on a space form. Let $\mathbb{E}^{3}$ be a simply-connected space form $\mathbb{R}^{3}, S^{3}$ or $\mathbb{H}^{3}$ and let $U$ be an open subset of $\mathbb{E}^{3}$ which is convex in the weak sense that any two points can be joined by a not necessarily minimal geodesic segment. Equivalently, the intersection of any maximal geodesic of $\mathbf{E}^{3}$ with $U$ must be connected. For example in $S^{3}$ both a small disk and the complement of its closure are convex in this sense as is the whole of $S^{3}$. In $\mathbb{R}^{3}$ and $\mathbb{H}^{3}$ this notion of convexity clearly coincides with the usual one. Note that through any two distinct points of $U$ there is a unique maximal geodesic $\gamma$ of $U$; if $U \subset S^{3}$ these may be closed; in all cases $\gamma$ is the intersection of a maximal geodesic of $\mathbb{E}^{3}$ with $U$.

Let $G_{U}$ be the space of all (oriented maximal) geodesics of $U$. This can be given the structure of a smooth real four-dimensional manifold as follows.

Given an oriented geodesic $\gamma \in G_{U}$ (see Figure 3), choose points $p$ and $q$ on $\gamma$, with $p$ preceeding $q$ with respect to the orientation and, if $\mathbb{E}^{3}=S^{3}$, with $\operatorname{dist}(p, q)<\pi$. (This ensures that $p$ and $q$ are not conjugate and there are no conjugate points between them.) Let $K$ and $L$ be slices at $p$ and $q$, that is, connected open subsets of planes orthogonal to $\gamma$ at $p$ and $q$. (In case $\mathbb{E}^{3}=S^{3}, K$ and $L$ are assumed to be sufficiently small that $\operatorname{dist}\left(p^{\prime} q^{\prime}\right)<\pi$, for all $p^{\prime} \in K, q^{\prime} \in L$.) Define a mapping $c_{K L}: K \times L \rightarrow G_{U}$

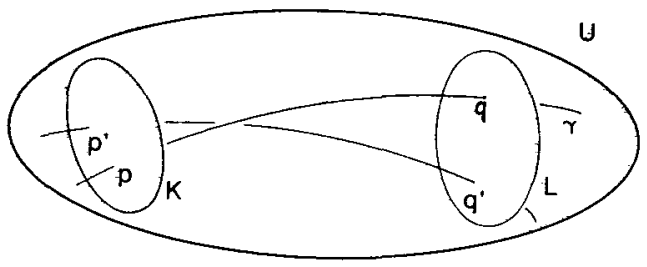

Figure 3 
by $\left(p^{\prime}, q^{\prime}\right) \rightarrow$ unique maximal geodesic through $p^{\prime}$ and $q^{\prime}$ with orientation consistent with that of $\gamma$. Then the collection of all $c_{K L}$ gives $G_{U}$ the structure of a smooth four-dimensional manifold. Note that $G_{U}$ is the open subset of $G_{\mathbf{E}^{3}}$ consisting of those $\gamma \in G_{\mathbf{E}^{3}}$ having non-empty intersection with $U ; G_{\mathbf{E}^{3}}$ is clearly Hausdorff, and thus so is $G_{U}$.

The tangent space $T_{\gamma} G_{U}$ may be identified with $T_{p} K \times T_{q} L$. Alternatively, recall that a Jacobi field $Y$ along $\gamma$ is a vector field along $\gamma$ which satisfies the equation

$$
\nabla_{X}^{2} Y+R(Y, X) X=0,
$$

where $X$ is the unit tangent to $\gamma, \nabla$ is the Levi-Civita connection on $\mathbb{E}^{3}$ and $R$ the curvature tensor. If $s \rightarrow \gamma_{s}$ is a curve of geodesics in $\mathbb{E}^{3}$ with $\gamma_{0}=\gamma$, then $Y=d \gamma /\left.d s\right|_{s=0}$ is a Jacobi field. By a normal Jacobi field we mean one which is orthogonal to $X$ at all points. The normal component of any Jacobi field is a normal Jacobi field.

Given $(v, w) \in T_{p} K \times T_{q} L$, there is a unique normal Jacobi field along $\gamma$ with the values $v, w$ at $p, q$. We may thus identify $T_{\gamma} G_{U}$ with the space of normal Jacobi fields along $\gamma$. Now for any $x \in \gamma$, write $H_{x}$ for the normal space ("horizontal space") $\left(T_{x} \gamma\right)^{\perp}$; thus $H_{p}=T_{p} K, H_{q}=T_{q} L$. Give $H_{x}$ the orientation which together with the orientation of $\gamma$ gives the standard orientation of $\mathbb{E}^{3}$. Define $J=J_{x}: H_{x} \rightarrow H_{x}$ to be rotation through $+\pi / 2$. In terms of the vector cross product on $T_{x} \mathbb{E}^{3} \cong \mathbb{R}^{3}$ this can be written $J(Y)=X \times Y$, where $X$ is the unit positive tangent to $\gamma$. As a consequence of constant curvature (see [15]), if $Y$ is a normal Jacobi field along $\gamma$ so is $J Y$. Thus $J$ defines an almost complex structure on $G_{U}$. This is integrable (see [15]). Note that under the identification of $T_{\gamma} G_{U}$ with $T_{p} K \times T_{q} L \cong H_{p} \times H_{q}, J$ corresponds to the product $\left(J_{p}, J_{q}\right)$.

(B) Conformal foliations by geodesics. Again, let $U$ be a convex (in the weak sense of Section A) open subset of $\mathbb{E}^{3}=\mathbb{R}^{3}, S^{3}$ or $\mathbb{H}^{3}$ and let $\mathscr{F}$ be an oriented $C^{\infty}$ foliation by geodesics. (Note that if $U$ is simply-connected, in particular if $U$ is convex in the usual sense, any foliation can be oriented by transporting orientations along paths.)

\section{LeMmA 2.1. The leaves of $\mathscr{F}$ are maximal geodesics of $U$.}

REMARK. This result holds for an arbitrary open subset $U$ of $\mathbb{E}^{3}$ whether convex or not.

Proof. Suppose not. Then some leaf contains an open interval, up to $x \in U$ say, of a maximal geodesic $\gamma$ but does not contain $x$. But then $x$ 


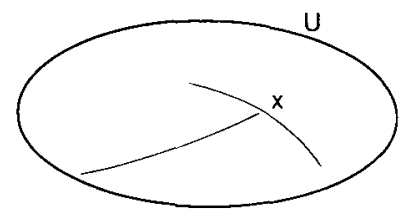

Figure 4

must lie on some other leaf and it is clear that $\mathscr{F}$ cannot be a foliation near $x$. (See Figure 4.)

Note that from the explicit description of geodesics on $\mathbb{E}^{3}$, any foliation by geodesics has trivial holonomy.

Now let $N$ be the set of leaves ("leaf space") of $\mathscr{F}$. Since each leaf is an oriented maximal geodesic of $U, N$ is naturally a subset of $G_{U}$, that is, we have an inclusion mapping $i: N \rightarrow G_{U}$.

LEMMA 2.2. $N$ is a two-dimensional smooth submanifold of $G_{U}$.

Proof. Let $\gamma \in N$, and let $p, q, K, L$ be as in Section A. Thus $c_{K L}: K \times L \rightarrow G_{U}$ gives a chart for $G_{U}$ near $\gamma$. Define a map $s$ from a neighbourhood $K^{\prime}$ of $p$ in $K$ to a neighbourhood $L^{\prime}$ of $q$ in $L$ ("sliding along the leaves") by $s(x)=$ the intersection of the leaf through $x$ with $L$. For a suitable choice of $K^{\prime}$ and $L^{\prime}, s$ is a diffeomorphism. Now in the chart $c_{K L}, N \cap c_{K L}\left(K^{\prime} \times L^{\prime}\right)$ is the graph of $s$, that is, $N \cap c_{K L}\left(K^{\prime} \times L^{\prime}\right)=$ $c_{K L}\left(\left\{(x, s(x)) \in K \times L: x \in K^{\prime}\right\}\right)$. This establishes the lemma.

Remark 2.3. (i) It follows that charts for the smooth two-dimensional manifold $N$ are given as follows. Let $\gamma \in N$ and choose a point $x \in \gamma$. Let $K$ be a slice for $\mathscr{F}$ at $x$, that is, $K$ is a connected open subset of a plane which cuts $\gamma$ orthogonally and is sufficiently small that leaves through points of $K$ intersect $K$ transversally. Then $c_{K}: K \rightarrow N$ given by $p^{\prime} \mapsto$ maximal geodesic of $U$ through $p^{\prime}$, gives charts for $N$. In fact $N$ is oriented by this atlas. Note that the tangent space $T_{y} N$ may be identified with $T_{x} K$ or with the space of basic (Section A) horizontal vector fields along $\gamma$. Evaluation at $x$ gives an isomorphism of the latter with the former.

(ii) We can consider $N$ as the quotient space $U / \sim$, where $x \sim y$ if and only if they lie on the same leaf. It is easy to check that the quotient topology agrees with the topology of $N$ as a submanifold of $G_{U}$. As in (i) above, $N$ may then be given the structure of a smooth oriented two-dimensional manifold without reference to the inclusion $i: N \rightarrow G_{U} ; c f$. [4].

We now identify conformal foliations by the following 
Proposition 2.4. $N$ is a complex submanifold of $G_{U}$, that is, the inclusion map $i: N \rightarrow G_{U}$ is holomorphic if and only if $\mathscr{F}$ is a conformal foliation.

Proof. Let $\gamma \in N$. It is clear that $i * T_{\gamma} N$ is the subset of those normal Jacobi fields along $\gamma$ which are basic. Thus $i$ is holomorphic if and only if $J$ maps basic horizontal vector fields to basic horizontal vector fields. But by Proposition 1.12, this is equivalent to $\mathscr{F}$ being a conformal foliation.

In particular we have

COROLLARY 2.5. If $F$ is an oriented conformal foliation by geodesics of a convex subset $U$ of $\mathbb{E}^{3}$, the leaf space of $\mathscr{F}$ is a Riemann surface.

REMARK 2.6. (i) The charts described in Remark 2.3 (i) are complex charts for the leaf space.

(ii) An alternative proof of Proposition 2.4 is to note that the differential of the "sliding along the leaves map" $s: K \rightarrow L$ (see proof of Lemma 2.2) is parallel transport along the leaves (see Section 1C) and this respects $J$ if and only if the foliation $\mathscr{F}$ is conformal.

(C) Relationship between conformal foliations by geodesics and harmonic morphisms; examples. As a special case of Proposition 1.10 and Remark 1.11 we have

LEMMA 2.7. Let $\phi: M^{3} \rightarrow N^{2}$ be a submersive harmonic morphism from an oriented three-dimensional Riemannian manifold to a Riemann surface. Then the foliation associated to $\phi$, that is, the foliation $\mathscr{F}$ whose leaves are the connected components of the fibres of $\phi$, is a conformal foliation of $M^{3}$ by geodesics. Further $F$ may be given a canonical orientation.

The canonical orientation is obtained as follows. Orient each horizontal space $H_{x}$ so that $d \phi_{x} \mid H_{x}$ is orientation preserving. Then orient each vertical space $V_{x}(=$ tangent space to the leaf through $x)$ such that the orientation of $H_{x}$ and $V_{x}$ together give the orientation of $M^{3}$.

EXAMPLes 2.8 ( $c f$. Examples 1.2). (i) Corresponding to orthogonal projection $\phi: \mathbb{R}^{3} \rightarrow \mathbb{R}^{2}$ is the conformal (in fact, Riemannian) foliation of $\mathbb{R}^{3}$ by vertical straight lines.

(ii) Corresponding to the Hopf map $\phi: S^{3} \rightarrow S^{2}$ is the conformal (in fact, Riemannian) foliation of $S^{3}$ by great circles. 
(iii) Corresponding to orthogonal projection $\phi: \mathbb{H}^{3} \rightarrow \mathbb{H}^{2}$ is the conformal (not Riemannian) foliation of $\mathbb{H}^{3}$ by hyperbolic geodesics depicted in Figure 2(a).

(iv) Corresponding to projection to the plane at infinity $\phi: \mathbb{H}^{3} \rightarrow \mathbb{C}$ is the conformal (not Riemannian) foliation of $\mathbb{H}^{3}$ by hyperbolic geodesics depicted by Figure 2(b). In the half-space model of $\mathbb{H}^{3}$ this foliation is the foliation of the upper half-space by vertical straight lines.

We shall refer to the above four foliations as the standard foliations of the simply-connected space forms $\mathbb{E}^{3}=\mathbb{R}^{3}, S^{3}$ and $\mathbb{H}^{3}$. Our main result (Corollary 3.4) is that, up to isometry of $\mathbb{E}^{3}$, these are the only conformal foliations of these spaces by geodesics. However other conformal foliations by geodesics can be defined on open subsets of these spaces. In particular corresponding to the radial projections of Examples 1.7 we have the following conformal foliations by geodesics.

EXAMPLES 2.9. (i) The conformal foliation of $\mathbb{R}^{3} \backslash\{0\}$ by radii, that is, half-lines from the origin.

(ii) The conformal foliation of $S^{3} \backslash\{(1,0,0,0),(-1,0,0,0)\}$ by half greatcircles through $( \pm 1,0,0,0)$.

(iii) The conformal foliation of $\mathbb{H}^{3} \backslash\{0\}$ by radii, that is, geodesics from the origin.

As a converse to Lemma 2.7 we have

LEMMA 2.10. Given an oriented conformal foliation $\mathscr{F}$ by geodesics of an open subset $U$ of $\mathbb{E}^{3}=\mathbb{R}^{3}, S^{3}$ or $\mathbb{H}^{3}$, convex in the weak sense of Section $2 A$, let $N$ be its leaf space and let $\pi: U \rightarrow N$ be the natural projection. Then $\pi$ is a (submersive and surjective) harmonic morphism with connected fibres.

Proof. Clearly $\pi$ is horizontally conformal and has geodesic fibres and is therefore a harmonic morphism by Theorem 1.1.

Say that two nonconstant submersive surjective harmonic morphisms to Riemann surfaces $\phi: M^{3} \rightarrow N^{2}$ and $\phi^{\prime}: M^{3} \rightarrow N^{\prime 2}$ are equivalent if $\phi^{\prime}=\zeta \circ \phi$ for some biholomorphic map $\zeta: N^{2} \rightarrow N^{\prime 2}$. Then combining Lemmas 2.7 and 2.10 we have

Proposition 2.11. Let $U$ be an open subset of $\mathbb{E}^{3}=\mathbb{R}^{3}, S^{3}$ or $\mathbb{H}^{3}$, convex in the weak sense of Section $2 \mathrm{~A}$. The above constructions give a one-to-one correspondence between oriented conformal foliations of $U$ by geodesics and equivalence classes of submersive surjective harmonic morphisms with connected fibres of $U$ to a Riemann surface. 
Proof. Clearly equivalent harmonic morphisms give the same foliation. Now let $\phi: U \rightarrow N^{2}$ be a submersive surjective harmonic morphism with connected fibres. Let $\mathscr{F}$ be the associated foliation. Then up to biholomorphic equivalence, $N^{2}$ is the leaf space of $\mathscr{F}$ and $\phi$ the natural projection onto it and the Proposition is proved.

Remark 2.12. (i) A version for codomains $N$, two-dimensional Riemannian manifolds not necessarily orientable, is true if we replace biholomorphic by bi-conformal (that is, conformal diffeomorphism) and drop the requirement that the foliations be oriented.

(ii) Since any harmonic morphism to a Riemann surface is $C^{\omega}$ (for any harmonic map is) any smooth conformal foliation by geodesics of an open subset $U$ of $\mathbb{E}^{3}=\mathbb{R}^{3}, S^{3}$ or $\mathbb{H}^{3}$ is actually $C^{\omega}$ for it is (locally) given by a (submersive) harmonic morphism.

If $U$ is the whole of $\mathbb{E}^{3}$ we can say more about $\pi$ :

Proposition 2.13. Let $\mathbb{E}^{3}=\mathbb{R}^{3}, S^{3}$ or $\mathbb{H}^{3}$ and let $\mathscr{F}$ be a $C^{r}$ foliation of $\mathbb{E}^{3}$ by geodesics $(r=0,1, \ldots, \infty, \omega)$. Then the natural projection $\pi$ : $\mathbb{E}^{3} \rightarrow N$ onto the leaf space is a principal $C^{r}$ bundle with fibre $I=\mathbb{R}$ if $\mathbb{E}^{3}=\mathbb{R}^{3}$ or $\mathbb{H}^{3}, I=S^{1}$ if $\mathbb{E}^{3}=S^{3}$.

Further if $\mathbb{E}^{3}=\mathbb{R}^{3}$ or $\mathbb{H}^{3}, N$ is contractible and $\pi$ is globally trivial, that is, there is a $C^{r}$ diffeomorphism $\sigma$, isometric on the second factor, such that the following diagram commutes:

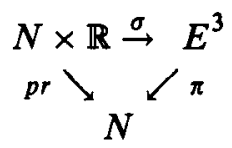

Proof. Orient $\mathscr{F}$. Let $p \in \mathbb{E}^{3}$ and let $K$ be a slice at $p$. Then $K$ may be identified via a chart with an open subset of the leaf space $N$. Let $\sigma: K \times I \rightarrow$ $\mathbb{E}^{3}$ be the flow along the leaves, that is, $\sigma(x, t)=$ the point at a distance $t$ from $x$ along the leaf through $x$. Then $\sigma$ is a $C^{r}$ diffeomorphism and so $\pi$ is locally trivial. In fact, $\sigma$ is isometric on the second factor, showing that $\pi$ is actually a principal fibre bundle with structure group $I$.

If now $\mathbb{E}^{3}=\mathbb{R}^{3}$ or $\mathbb{H}^{3}$, the homotopy exact sequence of $\pi$ (for example [19]) shows that the surface $N$ has $\pi_{1}(N)=\pi_{2}(N)=0$. It is therefore contractible. It follows (for example, [12, Proposition II.3.2]) that $\pi$ has a global section and is therefore globally trivial.

REMARK. We have not insisted that $\mathscr{F}$ is conformal.

As a consequence of Proposition 2.13 we can translate the Bernstein theorems [4, Theorems 4.1 and 5.1] into the following theorems for foliations: 
THEOREM 2.14. Let $\mathscr{F}$ be a smooth conformal foliation by geodesics of $\mathbb{R}^{3}$. Then, up to isometry of $\mathbb{R}^{3}, \mathscr{F}$ is the standard foliation of Example 2.8(i).

THEOREM 2.15. Let $F$ be a smooth conformal foliation be geodesics of $S^{3}$. Then, up to isometry of $S^{3}, F$ is the standard foliation of Example 2.8(ii).

For the corresponding result on $\mathbb{H}^{3}$, see Theorem 3.3.

(D) Representation of harmonic morphisms by the inclusion map $i$ and examples. Let $\mathbb{E}^{3}=\mathbb{R}^{3}, S^{3}$ or $\mathbb{H}^{3}$. Let $U \subset \mathbb{E}^{3}$ be a convex open set in the weak sense of Section 2A and let $\phi: U \rightarrow N$ be a submersive surjective harmonic morphism to a Riemann surface with connected fibres, or equivalently (Proposition 2.11), an oriented conformal foliation $\mathscr{F}$ of $U$ by geodesics. Then as in Proposition 2.4 we have a holomorphic inclusion map $i: N \rightarrow G_{\mathbf{E}^{3}}$ of a complex submanifold which describes the location of each fibre. Conversely, given a holomorphic inclusion map $i: N \rightarrow G_{\mathbf{E}^{3}}$ of a one-dimensional complex submanifold $N$, we obtain an "oriented conformal distribution" of geodesics $\{i(y): y \in N\}$ which may or may not form a foliation. However if $U$ is an open subset of $\mathbb{E}^{3}$ such that

(a) each $x \in U$ lies on a unique geodesic $i(y)$ for some $y \in N$,

(b) for $y_{1}, y_{2} \in N, y_{1} \neq y_{2}$, the geodesics $i\left(y_{1}\right), i\left(y_{2}\right)$ do not interest in $U$,

(c) the distribution $\{i(y): y \in N\}$ has no envelope points (that is, infinitesimally close geodesics do not intersect), then the distribution does define a foliation $\mathscr{F}$ on $U$ by geodesics. If $U$ is convex, by Proposition 2.11 this foliation corresponds to a harmonic morphism $\phi: U \rightarrow N$. In particular the inclusion map $i: N \rightarrow G_{\mathbf{E}^{3}}$ here plays the role of the generalized harmonic morphism of [4].

For examples of conformal foliations and the related inclusion maps for $\mathbb{E}^{3}=\mathbb{R}^{3}$ and $S^{3}$, we refer to [4]. The case $\mathbb{E}^{3}=\mathbb{H}^{3}$ will be discussed in Section 3.

(E) Factorization of harmonic morphisms. We first discuss a map related to the holomorphic inclusion map $i: N \rightarrow G_{U}$. Let $U$ be an arbitrary open subset of $\mathbb{E}^{3}$ and let $\mathscr{F}$ be an oriented foliation of $U$ by geodesics. We define a mapping $I: U \rightarrow G_{\mathbf{E}^{3}}$ by $x \mapsto$ leaf through $x$.

Proposition 2.16. If $\mathscr{F}$ is conformal, the components of $I$ with respect to any complex chart are harmonic morphisms. That is, let $c=\left(c_{1}, c_{2}\right): V \rightarrow \mathbb{C}^{2}$ be a complex chart on an open subset $V$ of $G_{\mathbf{E}^{3}}$; then the maps $c_{i} \circ I: U \supseteq$ $I^{-1}(V) \rightarrow \mathbb{C}$ are harmonic morphisms. Furthermore the real and imaginary 
parts of the components of $I, \operatorname{Re}\left(c_{i} \circ I\right), \operatorname{Im}\left(c_{i} \circ I\right): I^{-1}(V) \rightarrow \mathbb{R}$ are harmonic functions.

Proof. Let $p \in U$ and let $U_{p}$ be a convex neighbourhood of $p$. Then we may form the leaf space $N$ of $\mathscr{F} \mid U_{p}$ as in Section 2B and by Lemma 2.10 the natural projection $\pi: U_{p} \rightarrow N$ is a harmonic morphism. Also by Proposition 2.4 the inclusion map $i: N \rightarrow G_{\mathbf{E}^{3}}$ is holomorphic. Now, on $U_{p}, I=i \circ \pi$, so $c_{i} \circ I=\left(c_{i} \circ i\right) \circ \pi$, which exhibits $c_{i} \circ I$ as the composition of a holomorphic map $c_{i} \circ i$ with the harmonic morphism $\pi$. As in Corollary 1.2(vii), this composition is a harmonic map and therefore so are its real and imaginary parts.

We now wish to study harmonic morphisms $\phi$ which may not be submersions, nor have connected fibres. We shall show that we can still associate a foliation to $\phi$. So let $\phi: U \rightarrow N^{2}$ be a non-constant harmonic morphism from a convex open subset $U$ of a three-dimensional Riemannian manifold to a Riemann surface, and let $K$ denote its critical set (Section 1A).

LeMMA 2.17. $I: U \backslash K \rightarrow G_{\mathbf{E}^{3}}$ has a unique continuous extension to $U$ and the fibres of $\phi$ give a $C^{0}$ foliation of $U$ by geodesics with the connected component of the fibre $\phi^{-1}(p)$ through $p$ given by $I(p)$ for all $p \in U$.

Proof. This is proved in [5] for $\mathbb{E}^{3}=\mathbb{R}^{3}$. Since the notions of capacity, polar set used in that proof are independent of the Riemannian metric (cf. [7]), the same proof works for any three-dimensional Riemannian manifold.

For an alternative proof using the fact that the symbol of a harmonic morphism is a harmonic morphism [9] see [2].

Now let $U$ be an open subset of a space form $\mathbb{E}^{3}=\mathbb{R}^{3}, S^{3}$ or $\mathbb{H}^{3}$. Then we can say more:

Theorem 2.18. Let $\mathscr{F}$ be a $C^{0}$ foliation of $U$ by geodesics which is $C^{\infty}$ and conformal on $U \backslash K$ where $K$ is polar in $U$. Then $\mathscr{F}$ is $C^{\infty}$ (in fact $C^{\omega}$ ) and conformal on $U$.

Proof. Let $I: U \rightarrow G_{\mathbf{E}^{3}}$ be the mapping $x \mapsto$ leaf through $x$. Then $I$ is $C^{0}$ on $U$ and $C^{\infty}$ on $U \backslash K$. Thus its four real components with respect to a complex chart are $C^{0}$ on $U$ and, by Proposition $2.17, C^{\infty}$ and harmonic on $U \backslash K$. By a well-known extension property for harmonic functions [14, Corollary 7.8] these functions are $C^{\infty}$ and harmonic on $U$, thus $I$ is $C^{\infty}$ on $U$. It follows that $\mathscr{F}$ is $C^{\infty}$ on $U$ and therefore clearly conformal. It is in fact $C^{\omega}$ either by noting any harmonic function on a $C^{\omega}$ manifold is $C^{\omega}$ or by Remark 2.12 (ii). 
This theorem allows us to factorize nonsubmersive harmonic morphisms.

THEOREM 2.19. Let $\phi: U \rightarrow N^{2}$ be a nonconstant harmonic morphism from a convex open subset of $\mathbb{E}^{3}=\mathbb{R}^{3}, S^{3}$ or $\mathbb{H}^{3}$ to a two-dimensional Riemannian manifold. Then $\phi$ factors as $\phi=\zeta \circ \pi$, where $\pi: U \rightarrow \widetilde{N}$ is a submersive harmonic morphism onto a Riemann surface with connected fibres and $\zeta: \widetilde{N} \rightarrow N$ is a weakly conformal map.

Proof. By Lemma 2.18 and Theorem 2.19, the components of the fibres of $\phi$ give a $C^{\infty}$ conformal foliation $\mathscr{F}$ of $U$. This can be oriented. As in Section $2 \mathrm{~A}$, especially Corollary 2.5 , we can form the leaf space $\tilde{N}$, a Riemann surface and the natural projection $\pi: U \rightarrow \widetilde{N}$ is a submersive harmonic morphism with connected fibres (Lemma 2.10). Since the leaves of $\mathscr{F}$ are components of the fibres of $\phi, \phi$ factors as $\zeta \circ \pi$ for some $\zeta: \tilde{N} \rightarrow N$. As in Remark 2.3, local $C^{\infty}$ charts for $\tilde{N}$ are given by slices $K$ of $\mathscr{F}$ showing $\zeta$ is locally $\left.\phi\right|_{K}$ and is therefore smooth. Since $\phi$ and $\pi$ are both horizontally (weakly) conformal, $\zeta$ must clearly be weakly conformal.

\section{Harmonic morphisms and conformal foliations by geodesics of $\mathbb{H}^{3}$}

In this section we study harmonic morphisms and conformal foliations by geodesics defined on $\mathbb{H}^{3}$. Firstly we describe the space of geodesics on $\mathbb{H}^{3}$, then we show how to represent a holomorphic inclusion map $i: N \rightarrow G_{\mathbf{H}^{3}}$ by a pair of meromorphic functions $f, g$. Then by studying the conditions on $f, g$ for a foliation defined globally on $\mathbb{H}^{3}$ we show our main result that, up to isometry, there are only two conformal foliations by geodesics defined on the whole of $\mathbb{H}^{3}$ (Theorem 3.3) with consequences for harmonic morphisms (Theorem 3.5).

Recall that in the Poincare model, three dimensional hyperbolic space $\mathbb{H}^{3}$ is identified with the open unit ball $D^{3}=\left\{x \in \mathbb{R}^{3}:|x|<1\right\}$ and the maximal geodesics are the arcs of great circles whose closures meet the boundary $\partial D^{3}$ orthogonally. We thus have a bijection $b: G_{\mathbf{H}^{3}} \rightarrow S^{2} \times S^{2} \backslash \Delta$ from the space $G_{\mathbf{H}^{3}}$ of maximal oriented geodesics to the product of spheres minus the diagonal $\Delta=\left\{(x, y) \in S^{2} \times S^{2}: x=y\right\}$, given by $b(\gamma)=(x, y)$ where $x$ and $y$ are the beginning and endpoints of the arc $\gamma$. We shall refer to $\gamma$ as the geodesic (with endpoints) $(x, y)$.

Now if $Y$ is a Jacobi field (Section 2A) along a geodesic $\gamma, Y$ extends smoothly to $x$ and $y$ and from our description of the tangent space to the space of geodesics (Section 2A) we see that the differential of $b$ at $\gamma \in G_{\mathbf{H}^{3}}$ 


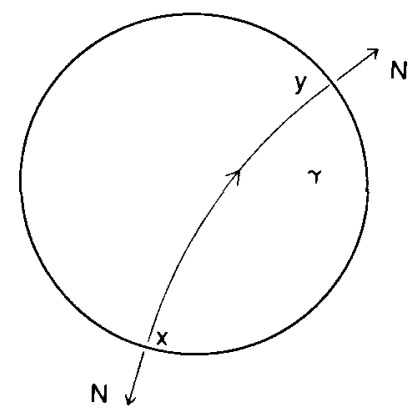

Figure 5

is the map $T_{y} G_{\mathbf{H}^{3}} \rightarrow T_{x} S^{2} \times T_{y} S^{2}, Y \mapsto\left(Y_{x}, Y_{y}\right)$. Giving $G_{\mathbf{H}^{3}}$ the almost complex structure $Y \rightarrow J Y=X \times Y$, where $X$ is the unit positive tangent to $\gamma$ (see Section 2A) and giving $S^{2}$ its standard almost complex structure $J$ defined by $J Y=N \times Y$, where $N$ is the unit outward normal, we see that $J Y \mapsto\left(-J Y_{x}, J Y_{y}\right)$, the minus sign because at $x, N$ and nearby unit positive tangents are oppositely sensed whereas at $y$ they are in the same sense. (See Figure 5.)

Let $S^{2}$ denote the two-sphere with its standard almost complex structure $J$ and let $\overline{S^{2}}$ denote the two-sphere with the "conjugate" almost complex structure $-J$. Then we have proved

LEMMA 3.1. The bijection $b: G_{\mathbf{H}^{3}} \rightarrow \overline{S^{2}} \times S^{2} \backslash \Delta$ is biholomorphic.

Now let $\mathscr{F}$ be an oriented conformal foliation by geodesics on a convex subset $U$ of $\mathbb{H}^{3}$. (Note that since a convex subset of $\mathbb{H}^{3}$ is simply connected any conformal foliation by geodesics can be oriented.) Denote its leaf space (Section 2B) by $N$ : and let $\pi: U \rightarrow N$ be the natural projection, that is, for $x \in U, \pi(x)=$ leaf through $x$. We shall call $\pi^{-1}(y) \quad(y \in N)$ the leaf (or geodesic) parametrized by $y$. Then as in Proposition 2.4 we have a holomorphic inclusion map $i: N \rightarrow G_{\mathbf{H}^{3}}$. Define maps $F, G: N \rightarrow S^{2}$ by

$$
b(i(y))=(F(y), G(y)),
$$

for each $y \in N$. That is, $(F(y), G(y))$ are the endpoints of the geodesic parametrized by $y \in N$. Note $F(y) \neq G(y)$ for all $y \in N$. Further write $\bar{f}=\sigma \circ F$ and $g=\sigma \circ G$, where $\sigma: S^{2} \rightarrow \mathbb{C} \cup\{\infty\}$ is stereographic projection and ${ }^{-}$denotes complex conjugation on $\mathbb{C} \cup\{\infty\}$. Then by Lemma 3.1, $f, g$ : $N \rightarrow \mathbb{C} \cup\{\infty\}$ are meromorphic. Thus to an oriented conformal foliation by geodesics of $U \subset \mathbb{H}^{3}$ is associated a pair of meromorphic functions $f, g$ on the Riemann surface $N$ with $\overline{f(y)} \neq g(y)$ for $y \in N$. Conversely, given such a pair, if the resulting distribution $\{i(y): y \in N\}$ satisfies conditions (a), 


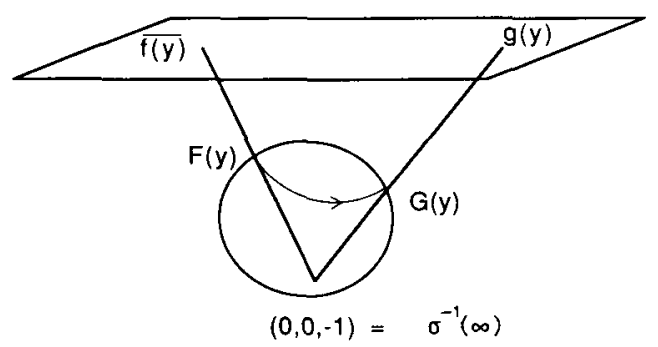

FIGURE 6

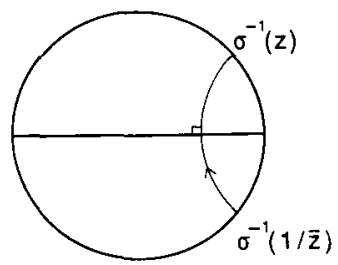

Figure 7

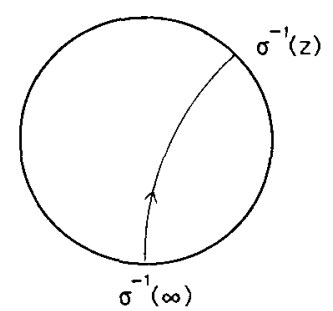

Figure 8

(b) and (c) of Section 2D it defines an oriented conformal foliation whose leaves are the oriented geodesics with end points $\left(\sigma^{-1}(\overline{f(y)}), \sigma^{-1}(g(y))\right)$. (See Figure 6.)

EXAMPLES 3.2. (i) Let $N=D^{2}, f(z)=1 / z, g(z)=z$. Then the geodesics run between points $F(z)=\sigma^{-1}(1 / \bar{z})$ and $G(z)=\sigma^{-1}(z)$ which are symmetrically placed with respect to the horizontal plane $x_{3}=0$ of $\mathbb{H}^{3}=D^{3}$ which they cross orthogonally. This $(f, g)$ thus corresponds to the standard foliation of Example 2.8(iii). (See Figure 7.)

(ii) Let $N=\mathbb{C}, f(z)=\infty, g(z)=z$. Then the geodesics run from the "South Pole" $(-1,0,0)$ of $S^{2}$ to the variable point $\sigma^{-1}(z)$ of $S^{2}$. This $(f, g)$ thus corresponds to the standard foliation of Example 2.8(iv). (Note that $f(z)=z, g(z)=\infty$ corresponds to the same foliation with the opposite orientation.) (See Figure 8.) 


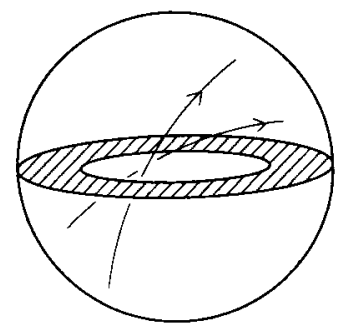

Figure 9

(iii) Let $N=D^{2}, f(z)=1 / z, g(z)=i z$. Then each geodesic runs from a point on a southern latitude to the point on the corresponding northern latitude but with longitude increased by $\pi / 2$. As $z \rightarrow \partial D^{2}$, the corresponding geodesic tends to a geodesic $\gamma_{\partial}$ joining points on the equator at an angle $\pi / 2$ apart. This gives a foliation which is not defined on the whole of $\mathbb{H}^{3}$ as the points of an annular region of the equatorial disc with outer boundary the equatorial circle and inner boundary the envelope of the geodesics $\gamma_{\partial}$ do not lie on any leaves. (See Figure 9.)

This foliation is similar to [4, Example 3.4 and 1, Example 4.2]. More generally, Examples (i) and (iii) are the extreme cases of a one-parameter family of conformal distributions by geodesic on $\mathbb{H}^{3}$ defined by $f(z)=1 / z$, $g(z)=e^{i \theta} z, 0 \leq \theta \leq \pi / 2$.

We now state and prove the main results of this chapter.

THEOREM 3.3. Let $\mathscr{F}$ be a smooth conformal foliation of $\mathbb{H}^{3}$ by geodesics. Then up to isometry of $\mathbb{H}^{3}, \mathscr{F}$ is one of the two standard foliations of $\mathbb{H}^{3}$ (Examples 2.8(iii) and (iv)).

Together with Theorems 2.14 and 2.15 this shows

COROllary 3.4. A smooth conformal foliation by geodesic of a simplyconnected space form $\mathbb{E}^{3}$ is, up to isometry of $\mathbb{E}^{3}$, one of the four standard foliations of Examples 2.8.

THEOREM 3.5. Let $\phi: \mathbb{H}^{3} \rightarrow N$ be a non-constant harmonic morphism to a two-dimensional Riemannian manifold. Then up to isometry of $\mathbf{H}^{3}, \phi$ is the composition $\phi=\zeta \circ \pi$, where $\pi$ is either orthogonal projection $\mathbb{H}^{3} \rightarrow \mathrm{H}^{2}$ (Example 1.2(iii)) or projection to the plane at infinity $\mathbb{H}^{3} \rightarrow \mathbb{C}$ (Example 1.2 (iv)) followed by a weakly conformal map to $N$. 
COROLlary 3.6. Let $\phi: \mathbb{H}^{3} \rightarrow N$ be a nonconstant harmonic morphism to an arbitrary Riemannian manifold. Then either

(i) $\operatorname{dim} N=1$ and $\phi$ is a harmonic map.

(ii) $\operatorname{dim} N=2$ and $\phi$ is as described in Theorem 3.5, or

(iii) $\operatorname{dim} N=3$ and $\phi$ is a local homothety.

Further $N$ is complete and $N=\mathbb{H}^{3}(c) / \Gamma$ where $\Gamma$ is a subgroup of the group of isometries of $\mathrm{H}^{3}(c)$ which acts freely and properly discontinuously and $\phi: \mathbb{H}^{3} \rightarrow \mathbb{H}^{3}(c) \rightarrow \mathbb{H}^{3}(c) / \Gamma$ is a homothety followed by the natural projection. (Here $\mathbb{H}^{3}(c)$ denotes $D^{3}$ with a metric $c>0$ times the standard one of Section $1 \mathbf{A}(\mathbf{i})$.)

Together with [4, Theorems 4.1 and 5.1] this completely describes all harmonic morphisms from a three-dimensional simply-connected space form $\mathbb{E}^{3}$. In particular, we have

COROLlaRY 3.7. Up to isometry of $\mathbb{E}^{3}$, a non-constant harmonic morphism from a simply-connected space form $\mathbb{E}^{3}$ to a two-dimensional Riemannian manifold $N$ must be one of the four standard examples (Examples 1.2) followed by a weakly conformal map to $N$.

To prove Theorem 3.3 we need an analytic criterion for intersecting geodesics. This is furnished by the classical notion of cross-ratio.

Given four complex numbers $z_{1}, w_{1}, z_{2}, w_{2} \in \mathbb{C} \cup\{\infty\}$, the cross-ratio is defined by the expression

$$
\left(z_{1}, w_{1} ; z_{2}, w_{2}\right)=\frac{z_{1}-z_{2}}{w_{2}-z_{2}} / \frac{z_{1}-w_{1}}{w_{2}-w_{1}}
$$

(see for example [6]).

Proposition 3.8. Consider a pair of geodesics $\gamma_{1}, \gamma_{2}$ in $\mathbb{H}^{3}$ with endpoints $\left(x_{1}, y_{1}\right),\left(x_{2}, y_{2}\right) \in S^{2} \times S^{2} \backslash \Delta$, which we identify under stereographic projection with pairs $\left(z_{1}, w_{1}\right),\left(z_{2}, w_{2}\right) \in(\mathbb{C} \cup\{\infty\}) \times(\mathbb{C} \cup\{\infty\}) \backslash \Delta$ (that is, $\left.z_{i}=\sigma\left(x_{i}\right), w_{i}=\sigma\left(y_{i}\right)\right)$. Then the geodesics $\gamma_{1}, \gamma_{2}$ intersect in $\mathbb{H}^{3}$ if and only if the cross-ratio $\left(z_{1}, w_{1} ; z_{2}, w_{2}\right)$ belongs to the subset $(0,1) \cup(1, \infty)$ of the real line $\mathbb{R} \subset \mathbb{C}$.

Proof. First note that the two geodesics with end points $\left(x_{1}, y_{1}\right),\left(x_{2}, y_{2}\right)$ intersect in $\mathbb{H}^{3}$ if and only if the corresponding (Euclidean) straight lines from $x_{1}$ to $y_{1}$ and from $x_{2}$ to $y_{2}$ intersect in the unit ball $D^{3}$. For as in Section $1 \mathrm{~A}$, the mapping $f(q)=q /\left(1+|q|^{2}\right)$ is a homeomorphism of the 


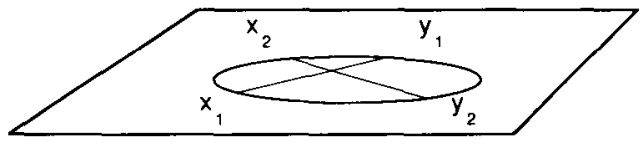

FIGURE 10

closed unit ball which sends the arc of a circle from $x_{1} \in S^{2}$ to $y_{1} \in S^{2}$ and orthogonal to $\partial D^{3}=S^{2}$ at $x_{1}$ and $y_{1}$, to the straight line from $x_{1}$ to $y_{1}$. Thus the two geodesics intersect if and only if the corresponding straight lines intersect. In this case the two lines determine a plane which intersects the boundary $\partial D^{3}=S^{2}$ in a circle containing the four points $x_{1}, y_{1}, x_{2}$, $y_{2}$ in alternating order, that is the pair $\left(x_{2}, y_{2}\right)$ separates the pair $\left(x_{1}, y_{1}\right)$ along the circle. (See Figure 10.)

Conversely, given four distinct points $x_{1}, y_{1}, x_{2}, y_{2}$, arranged in alternating order around a circle, then the corresponding straight lines joining them must intersect in $D^{3}$.

Now under stereographic projection, circles on $S^{2}$ are mapped to either circles or straight lines in the extended complex plane $\mathbb{C} \cup\{\infty\}$ and conversely. It is easily checked that the four distinct points $z_{1}, w_{1}, z_{2}, w_{2}$ lie on a circle or straight line in $\mathbb{C}$ in alternating order if and only if the cross-ratio $\left(z_{1}, w_{1} ; z_{2}, w_{2}\right)$ is real, positive and not equal to 1 .

Now let $\mathscr{F}$ be a smooth conformal foliation of $\mathbb{H}^{3}$ by geodesics and let $N$ denote its leaf space. Recall from Corollary 2.5 that $N$ is a Riemann surface and from Proposition 2.4 that we have a holomorphic inclusion map $i: N \rightarrow G_{\mathbf{H}^{3}}$.

LEMMA 3.9. The leaf space $N$ is biholomorphically equivalent to either the unit disc $D^{2}$ or the complex plane $\mathbb{C}$.

Proof. By Proposition 2.13, $N$ is contractible. The result follows by the Riemann mapping theorem (see for example [6]).

As described above the inclusion map $i$ for $\mathscr{T}$ is represented by a pair of meromorphic functions $f, g: N \rightarrow \mathbb{C} \cup\{\infty\}$ on the leaf space.

LEMMA 3.10. Either (a) $f$ and $g$ are both injective or (b) one of them is constant and the other is injective.

Proof. Suppose that both $f$ and $g$ are non-constant and suppose that $f$ is not injective; say $f\left(z_{0}\right)=f\left(z_{1}\right)$ for some $z_{0}, z_{1} \in N, z_{0} \neq z_{1}$. 


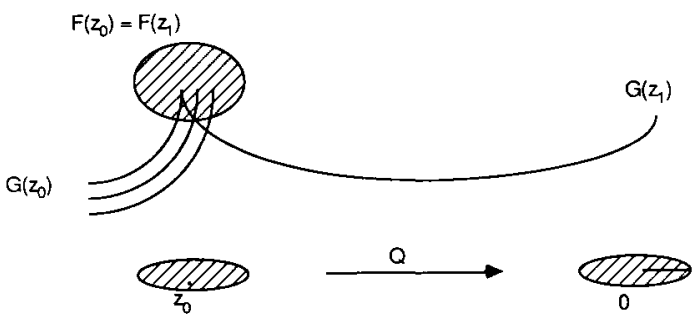

FIGURE 11

Then $g\left(z_{0}\right) \neq g\left(z_{1}\right)$ since the leaves parametrized by $z_{0}, z_{1}$ are distinct geodesics. Now it is intuitively clear that, as $z$ circles $z_{0}$ one of the geodesics $(\overline{f(z)}, g(z))$ will intersect the geodesic $\left(\overline{f\left(z_{1}\right)}, g\left(z_{1}\right)\right)$. (Here by the geodesic $(\overline{f(z)}, g(z))$ we mean the geodesic with endpoints $\left(\sigma^{-1}((\overline{f(z)}))\right.$, $\sigma^{-1}(g(z))$.) We prove this precisely by using the cross-ratio. (See Figure 11.)

We may assume $z_{0}$ is not a branch point of $f$, for if $z_{0}$ is a branch point, then close to $z_{0}$ we can find two points $w_{1}, w_{2}$ with $f\left(w_{1}\right)=f\left(w_{2}\right)$ and $w_{1}, w_{2}$ not branch points for $f$. We use the inverse function theorem on the cross-ratio

$$
Q(z)=\left(\vec{f}(z), g(z) ; \vec{f}\left(z_{1}\right), g\left(z_{1}\right)\right)=\frac{\vec{f}(z)-\bar{f}\left(z_{1}\right)}{g\left(z_{1}\right)-\bar{f}\left(z_{1}\right)} / \frac{\vec{f}(z)-g(z)}{g\left(z_{1}\right)-g(z)} .
$$

At $z=z_{0}$, by the hypothesis $f\left(z_{0}\right)=f\left(z_{1}\right)$,

$$
\begin{gathered}
Q\left(z_{0}\right)=0, \quad \frac{\partial Q}{\partial z}\left(z_{0}\right)=0, \\
\frac{\partial Q}{\partial \bar{z}}\left(z_{0}\right)=\frac{\left[g\left(z_{1}\right)-g\left(z_{0}\right)\right] \overline{f^{\prime}\left(z_{0}\right)}}{\left[g\left(z_{1}\right)-\bar{f}\left(z_{1}\right)\right]\left[\bar{f}\left(z_{0}\right)-g\left(z_{0}\right)\right]} \neq 0 .
\end{gathered}
$$

Therefore by the inverse function theorem, $Q$ is a local diffeomorphism at $z_{0}$ and maps a neighbourhood $U$ of $z_{0}$ onto a neighbourhood of $0 \in \mathbb{C}$. In particular, there is a one-parameter family of points $z \in U$ with $Q(z)$ real, positive and distinct from 1. By Proposition 3.8, the geodesics over such a point $z$ and $z_{1}$ intersect, a contradiction. By similar arguments, if both $f$ and $g$ are nonconstant $g$ must be injective.

Now suppose that one of $f, g$ is constant, say $g$. Then $f$ must be injective. For if $f\left(z_{0}\right)=f\left(z_{1}\right)$ for some $z_{0} \neq z_{1}$, then the leaves parametrized by $z_{0}, z_{1}$ are identical geodesics which is impossible.

Note that this lemma shows that neither $f$ nor $g$ can have any branch points. As before write $F=\sigma^{-1} \circ \bar{f}$ and $G=\sigma^{-1} \circ g$ where $\sigma: S^{2} \rightarrow \mathbb{C} \cup\{\infty\}$ is stereographic projection.

LEMMA 3.11. (i) $F(N)$ and $G(N)$ are disjoint subsets of $S^{2}$.

(ii) Closure $\{F(N) \cup G(N)\}=S^{2}$. 


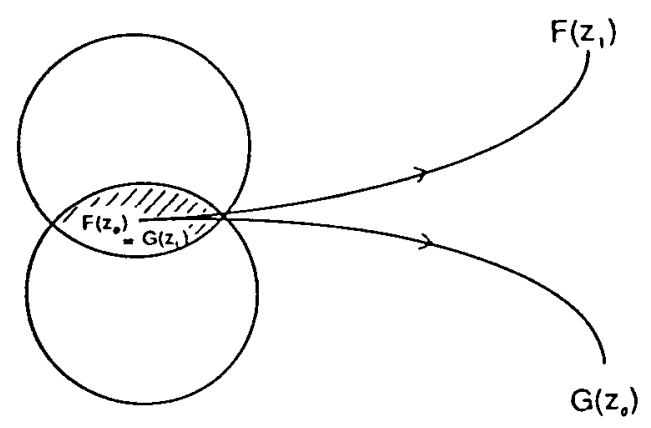

FIGURE 12

Proof. Let $z_{0}, z_{1} \in N, z_{0} \neq z_{1}$ and suppose that $F\left(z_{0}\right)=G\left(z_{1}\right)$. Then $f\left(z_{1}\right) \neq G\left(z_{0}\right)$, otherwise the leaves parametrized by $z_{0}$ and $z_{1}$ would be the same geodesic (with opposite orientations), which is impossible. Now, it is intuitively clear that as $z$ varies in a neighbourhood of $z_{0}$, the geodesic over $z$ must intersect the geodesic over $z_{1}$. As in Lemma 3.10 we prove this using the cross-ratio. (See Figure 12.)

Recall that $f$ and $g$ cannot both be constant; without loss of generality assume that $f$ is nonconstant. Consider the cross-ratio

$$
Q(z)=\frac{\bar{f}(z)-\bar{f}\left(z_{1}\right)}{g\left(z_{1}\right)-\bar{f}\left(z_{1}\right)} / \frac{\bar{f}(z)-g(z)}{g\left(z_{1}\right)-g(z)} .
$$

Then

$$
\begin{gathered}
Q\left(z_{0}\right)=1, \quad \frac{\partial Q}{\partial z}\left(z_{0}\right)=0, \\
\frac{\partial Q}{\partial \bar{z}}\left(z_{0}\right)=\frac{\overline{f^{\prime}\left(z_{0}\right)}\left[\bar{f}\left(z_{1}\right)-g\left(z_{0}\right)\right]}{\left[\bar{f}\left(z_{0}\right)-g\left(z_{0}\right)\right]\left[g\left(z_{1}\right)-\bar{f}\left(z_{1}\right)\right]} \neq 0 .
\end{gathered}
$$

Thus by the inverse function theorem, $Q$ maps a neighbourhood of $z_{0}$ diffeomorphically to a neighbourhood of $1 \in \mathbb{C}$. Therefore there are points $z \in N$ close to $z_{0}$ which map under $Q$ to a positive real number distinct from 1. By Proposition 3.8 the geodesic over such a $z$ intersects the geodesic $z_{1}$, a contradiction.

For the second part of the lemma suppose $F(N) \cup G(N)$ omits an open set $V$ of $S^{2}$. We may assume that this is a geodesic disc of $S^{2}$ bounded by a small circle on $S^{2}$. (See Figure 13.)

Recall that under the homeomorphism $A: D^{3} \rightarrow D^{3}$ of Section 1A, the geodesics of the Poincare model transform to straight lines (geodesics of the Beltrami model) with the same endpoints. But these straight lines have no points lying in the region between the plane of the circle bounding $V$ and 


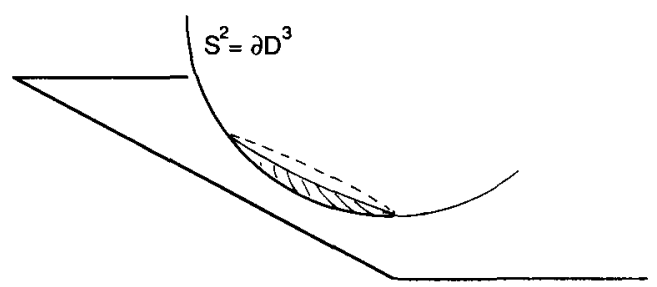

FIGURE 13

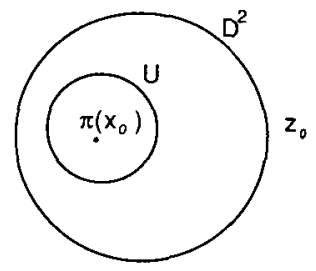

FIGURE 14

$\partial D^{3}$. Since $A$ is a homeomorphism, it follows that the union of the leaves of $F$ cannot be the whole of $\mathbb{H}^{3}$, a contradiction.

Recall from Lemma 3.9 that the leaf space of $\mathscr{F}$ must be biholomorphically equivalent to the disc $D^{2}$ or the complex plane $\mathbb{C}$. We consider these cases in turn.

CASE 1. Suppose that $N$ is biholomorphically equivalent to $D^{2}$. Then neither $f$ nor $g$ can be constant since, if for example, $g$ is constant say $g=\infty$, the leaves of the foliation are geodesics with endpoint at $\infty$ and we must therefore have the standard foliation of Example 3.2(ii). But this clearly has leaf space $S^{2} \backslash\{\infty\}=\mathbb{C}$, contradicting our assumption on $N$.

LEMMA 3.12. Let $z_{0} \in \partial D^{2}$. Then $\operatorname{dist}(F(z), G(z)) \rightarrow 0$ as $z \rightarrow z_{0}$ $\left(z \in D^{2}\right)$. (Here $\operatorname{dist}($,$) denotes standard distance between points of S^{2}$.)

Proof. We use the Beltrami model for $\mathbb{H}^{3}$. Suppose that the assertion of the lemma is false. Then there exists a sequence $\left(z_{n}\right)$ in $D^{2}$ with $z_{n} \rightarrow z_{0}$ such that $\operatorname{dist}\left(F\left(z_{n}\right), G\left(z_{n}\right)\right) \nrightarrow 0$. Since $S^{2}$ is compact, by extracting a subsequence if necessary, we may assume that $F\left(z_{n}\right) \rightarrow F_{0}$ and $G\left(z_{n}\right) \rightarrow$ $G_{0}$ for some $F_{0}, G_{0} \in S^{2}, F_{0} \neq G_{0}$. In particular the sequence of line segments (geodesics) $\pi^{-1}\left(z_{n}\right)$ with endpoints $\left(F\left(z_{n}\right), G\left(z_{n}\right)\right.$ ) approaches the line segment $\ell$ joining $F_{0}, G_{0}$. (See Figure 14.)

Let $x_{0} \in \ell$ and let $U$ be a neighbourhood of $\pi\left(x_{0}\right)=\ell$ in $D^{2}$ whose closure does not contain $z_{0}$. Then $\pi^{-1}(U)$ is an open set of $\mathbb{H}^{3}$ containing 
$\ell$. Now, since $\pi^{-1}\left(z_{n}\right)$ approaches $\ell$, for sufficiently large $n$, the geodesic $\pi^{-1}\left(z_{n}\right)$ must intersect $\pi^{-1}(U)$, therefore $z_{n} \in U$. But this contradicts $z_{n} \rightarrow z_{0} \notin \bar{U}$. The lemma follows.

To translate Lemma 3.12 into a statement about the meromorphic functions $f(z)$ and $g(z)$ we must ensure that neither tends to infinity as $z$ approaches the boundary $\partial D^{2}$. To do this apply an isometry of $\mathbb{H}^{3}$ such that $f$ attains the value $\infty$ at some point of $D^{2}$. For example, we can rotate $D^{3}$ about 0 to achieve this. Since by Lemma 3.10, $f(z)$ is injective, it is bounded away from $\infty$ as $z$ approaches $\partial D^{2}$, by Lemma 3.11 this is also true of $g(z)$. Then Lemma 3.12 implies that, for all $z_{0} \in \partial D^{2}$, $\overline{f(z)}-g(z) \rightarrow 0$ as $z \rightarrow z_{0}$.

LEMMA 3.13. The meromorphic functions $f, g: D^{2} \rightarrow \mathbb{C} \cup\{\infty\}$ extend to meromorphic functions on the extended complex plane $\mathbb{C} \cup\{\infty\}$. Further $f(z)=\overline{g(1 / \bar{z})}$ for all $z \in \mathbb{C} \cup\{\infty\}$.

Proof. Set $h(z)=\overline{f(1 / \bar{z})}$. Then $h$ is meromorphic on $\mathbb{C} \cup\{\infty\} \backslash \overline{D^{2}}$ and, for all $z_{0} \in \partial D^{2}, g(z)-\overline{f(z)}=g(z)-h(1 / \bar{z}) \rightarrow 0$ as $z \rightarrow z_{0}$. Furthermore $g$ and $h$ are analytic near the boundary $\partial D^{2}$ (see below). By Carlemann's extension principle [13, page 303] it follows that there is a meromorphic function on $\mathbb{C} \cup\{\infty\}$ extending $g$ and $h$. The lemma follows.

More explicitly, for each $z_{0} \in \partial D^{2}$, there is a ball $B\left(z_{0}\right)$ such that $g$ and $h$ are analytic in $B\left(z_{0}\right) \cap D^{2}$ and $B\left(z_{0}\right) \cap\left(\mathbb{C} \backslash \overline{D^{2}}\right)$ respectively. There is a bilinear transformation which sends $B\left(z_{0}\right) \cap \partial D^{2}$ to an interval of the real axis, for example, if $z_{0} \neq-1, w=i(z-1) /(z+1)$. Under such a transformation points $z, 1 / \bar{z}$ correspond to points $w, w$ and [13] may be applied to conclude that there is an analytic function on $B\left(z_{0}\right)$ extending $g$ and $h$. Such extensions for each $z_{0} \in \partial D^{2}$ glue together to give the required extension.

LEMMA 3.14. After an isometry of $\mathbb{H}^{3}$, the meromorphic functions $f, g$ are given by $f(z)=1 / z, g(z)=z$.

Proof. After a rotation of $\mathbb{H}^{3}$ we can assume that $f(0)=\infty$. Then by Lemma 3.10, $f$ has a simple pole at 0 and no other poles in $D^{2}$. By Lemma 3.11, $g$ has no poles in $D^{2}$. As in the arguments leading up to Lemma 3.13 neither $f$ nor $g$ can have a pole on $\partial D^{2}$. Since $g(z)=\overline{f(1 / \bar{z})}$ for all $z \in \mathbb{C} \cup\{\infty\}, g$ has no poles in $\mathbb{C} \cup\{\infty\} \backslash \overline{D^{2}}$, except for a simple pole at $\infty$. Therefore $g(z)=a_{0}+a_{1} z$ for some $a_{0}, a_{1} \in \mathbb{C}$ and $f(z)=\overline{a_{0}}+\overline{a_{1}} / z$. 
Now recall that any Möbius transformation interpreted as a transformation of $S^{2}=\partial D^{2}$ induces an isometry of $\mathbb{H}^{3}$. The Möbius transformation $z \mapsto$ $\left(z-a_{0}\right) / a_{1}$ transforms the pair $f(z), g(z)$ into $1 / z, z$ as required.

CASE 2. Suppose that $N$ is biholomorphically equivalent to $\mathbb{C}$.

LEMMA 3.15. After an isometry of $\mathbb{H}^{3}$, with respect to a suitable global complex coordinate $z$ on $N, f(z)=\infty, g(z)=z$ or $f(z)=z, g(z)=\infty$.

Proof. By Lemma 3.10, $f$ and $g$ cannot both be constant. Assume that $g$ is non-constant. Again by Lemma 3.10 the meromorphic map $g$ is injective so that its image $g(N) \subset \mathbb{C} \cup\{\infty\}$ omits precisely one point. By a rotation of $\mathbb{H}^{3}$ we may assume this point is $\infty$. Then $g: N \rightarrow \mathbb{C}$ is biholomorphic and so, with respect to a suitable complex coordinate $z$ (namely that given by the chart $g$ ),$g(z)=z$ for all $z \in N$. By Lemma 3.11, $f$ must be constant and equal to $\infty$. If $f$ is nonconstant we argue similarly.

Proof OF THEOREM 3.3. Let $\mathscr{F}$ be a conformal foliation by geodesics of $\mathbb{H}^{3}$. Orient $\mathscr{F}$. By Lemma 3.9 the leaf space $N$ is $D^{2}$ or $\mathbb{C}$. If $N=D^{2}$, Lemma 3.14 shows that, up to isometry of $\mathbb{H}^{3}, \mathscr{F}$ is the standard foliation of Example 3.2(i) (and 2.8(iii)). If $N=\mathbb{C}$, Lemma 3.15 shows that, up to isometry of $\mathbb{H}^{3}, \mathscr{F}$ is the standard foliation of Example 3.2(ii) (and 2.8(iv)).

Proof OF Theorem 3.5. Let $\phi: \mathbb{H}^{3} \rightarrow N$ be a nonconstant harmonic morphism to a two-dimensional Riemannian manifold. Then by Propositions 2.11 and $2.13, \phi=\zeta \circ \pi$ where $\pi: \mathbb{H}^{3} \rightarrow \widetilde{N}$ is a submersive harmonic morphism with connected geodesic fibres onto a Riemann surface and $\zeta: \widetilde{N} \rightarrow N$ is a weakly conformal map. By Theorem 3.3, the foliation associated to $\pi$ must be one of the standard ones (Examples 3.2(i) or (ii)). It follows (cf. Proposition 3.11) that $\pi$ is one of the two standard harmonic morphisms of $\mathbb{H}^{3}$ (Examples 1.2(iii) and (iv)).

Proof of Corollary 3.6. By Proposition 1.2, $n \leq 3$. The case $n=1$ follows from that Proposition and the case $n=2$ is Theorem 3.5.

For the case $n=3$ note first that by $[9$, Theorem 8] $\phi$ is homothetic. Then $N$ is complete, for let $p \in N$ and $x \in \phi^{-1}(p) \subset \mathbb{H}^{3}$. Then the exponential map $\exp _{p}^{N}: T_{p} N \rightarrow N$ is defined on all of $T_{p} N$, since any smooth geodesic emanating from $p$ is the image of a smooth geodesic emanating from $x$ in $\mathbb{H}^{3}$, which can be continued indefinitely. By the Hopf-Rinow Theorem [21, Theorem 2.8.8], $N$ is complete (and $\left.\phi\left(\mathbb{H}^{3}\right)=N\right)$. The result 
now follows from the theorem of Killing and Hopf [21, Corollary 2.4.10] describing complete connected Riemannian manifolds of constant curvature.

\section{Harmonic morphisms with isolated singularities}

In this chapter we study harmonic morphisms and conformal foliations by geodesics defined on connected open subsets of a simply-connected threedimensional space form $\mathbb{E}^{3}=\mathbb{R}^{3}, S^{3}$ or $\mathbb{H}^{3}$ which have isolated singularities. We show that any such conformal foliation must be a foliation by radii and correspondingly any harmonic morphism to a surface with an isolated singularity is locally a radial projection (see Theorems 4.1 and 4.2). Then we draw global conclusions showing that any conformal foliation by geodesics of $\mathbb{E}^{3}$ (or of an open subset $U$ of $\mathbb{E}^{3}$ ) with isolated singularities is, up to isometry, (the restriction of) a standard example (Examples 2.9) and correspondingly any harmonic morphism from $U$ to a surface with isolated singularities is, up to isometry, (the restriction of) one of the standard examples (Examples 1.7).

(A) Local theory. Let $B^{3}\left(p_{0}\right)$ denote a convex ball centre $p_{0}$ in a space form. By a radius we mean one of the two connected components $\gamma_{1}, \gamma_{2}$ of $\gamma \backslash\left\{p_{0}\right\}$, where $\gamma$ is a geodesic of $B^{3}\left(p_{0}\right)$ through $p_{0}$. Our main result is

THEOREM 4.1. Let $\mathscr{F}$ be a smooth conformal foliation of the deleted ball $B^{3}\left(p_{0}\right) \backslash\left\{p_{0}\right\}$ by geodesics. Then either $\mathscr{F}$ is the radial foliation, that is, the foliation with leaves given by the radii, or $\mathscr{F}$ extends to a smooth conformal foliation of $B^{3}\left(p_{0}\right)$.

THEOREM 4.2. Let $\phi: B^{3}\left(p_{0}\right) \backslash\left\{p_{0}\right\} \rightarrow N$ be a smooth harmonic morphism to a two-dimensional Riemannian manifold. Then either $\phi$ is radial projection $B^{3}\left(p_{0}\right) \backslash\left\{p_{0}\right\} \rightarrow S^{2}, x \mapsto x /|x|$ followed by a weakly conformal map $S^{2} \rightarrow N$ (and so $N=S^{2}$ or $\mathbb{R} P^{2}$ ), or $\phi$ extends to a smooth harmonic morphism $B^{3}\left(p_{0}\right) \rightarrow N$.

Here $x=\left(x_{1}, x_{2}, x_{3}\right)$ denotes normal coordinates centred on $p_{0}$ and, as usual, $|x|=\sqrt{ }\left(x_{1}^{2}+x_{2}^{2}+x_{3}^{2}\right)$. In these coordinates the radii are the half-lines emanating from the origin. For convenience we shall use these coordinates so that $B^{3}\left(p_{0}\right)$ becomes the ball $B^{3}$ with centre $0=(0,0,0)$.

To prove Theorem 4.1, we let $\mathscr{F}$ be a smooth conformal foliation by geodesics of the deleted ball $B^{3} \backslash\{0\}$. Then by Lemma 2.1 and the subsequent remark, the leaves of $\mathscr{F}$ are maximal geodesics of $B^{3} \backslash\{0\}$. These may either 
be radii or leaves avoiding 0 . Note that since $B^{3} \backslash\{0\}$ is simply connected $\mathscr{F}$ may be oriented; we assume this has been done.

Lemma 4.3. At least one radius is a leaf.

Proof. Choose a small ball centre $0, \widetilde{B}^{3} \subset B^{3}$. Then any leaf through a point of $\widetilde{\boldsymbol{B}}^{3} \backslash\{0\}$ must intersect the boundary $\partial \widetilde{\boldsymbol{B}}^{3}$. Suppose no radius is a leaf. Then the function $d: \partial \widetilde{B}^{3} \rightarrow[0, \infty)$ given by $d(x)=$ minimum distance from 0 of a point on the leaf through $x$, is continuous and does not attain the value 0 . Therefore it has a minimum value $\varepsilon>0$. But then no leaf goes inside the ball centre 0 , radius $\varepsilon$, in contradiction to the definition of foliation.

Now let $\gamma_{1} \subset B^{3} \backslash\{0\}$ be a radius which is a leaf and let $x_{0} \in \gamma_{1}$. Let $W$ be a slice for $\mathscr{F}$ at $x_{0}$ (that is, a connected open subset of a plane which cuts the leaf through $x_{0}$ orthogonally and is sufficiently small that leaves through points of $K$ cut $K$ transversally). We have two cases.

CASE 1. There is a sequence of points $x_{n} \in W$ with $x_{n} \rightarrow x_{0}$ such that the leaves through each $x_{n}$ are radii.

CASE 2. With $W$ replaced by a smaller slice if necessary, all the leaves through $W \backslash\left\{x_{0}\right\}$ avoid 0 .

LEMMA 4.4. In Case 1, the foliation is the radial foliation.

Proof. Let $G_{B^{3}}$ be the space of oriented maximal geodesics on $B^{3}$; this is a two-dimensional complex manifold; see Section 2A. Then [15] the subset of those oriented geodesics through 0 is a complex one-dimensional submanifold $\mathbb{P}^{1}$, biholomorphic to a complex line. Consider the map $I: B^{3} \backslash\{0\} \rightarrow G_{B^{3}}$ (cf. Section 2E). On any convex subset $U$ of $B^{3} \backslash\{0\}, I$ factors as the natural projection $U \rightarrow N_{U}$ onto the leaf space of $\left.\mathscr{F}\right|_{U}$, followed by the holomorphic inclusion map $i: N_{U} \rightarrow G_{B^{3}}$ of the complex submanifold $N_{U}$. Since $B^{3} \backslash\{0\}$ is covered by a finite number of convex open sets, it follows that the image of $I$ is an immersed complex submanifold. But for the points $x_{n}, I\left(x_{n}\right) \in \mathbb{P}^{1}$. Thus the intersection of $\operatorname{Im}(I)$ and $\mathbb{P}^{1}$ has an accumulation point on $\mathbb{P}^{1}$. It follows by analyticity that $\operatorname{Im}(I) \subset \mathbb{P}^{1}$, so that all leaves of $\mathscr{F}$ are radii as required.

We now turn to Case 2. If $\gamma_{1}$ is a radius, by the opposite radius $\gamma_{2}$ we mean the radius $\gamma_{2}$ such that $\gamma=\gamma_{1} \cup\{0\} \cup \gamma_{2}$ is a geodesic through 0 .

LEMma 4.5. In Case 2, let $\gamma_{1}$ be a radius which is a leaf. Then the opposite radius $\gamma_{2}$ is also a leaf with orientation such that $\gamma_{1} \cup\{0\} \cup \gamma_{2}$ is oriented. 
Proof. Let $I: B^{3} \backslash\{0\} \rightarrow G_{B^{3}}$ be the map $x \mapsto$ oriented maximal geodesic containing the fibre through $x$ (cf. Section 2E). Let $y \in \gamma_{2}$. Let $x_{0} \in \gamma_{1}$ and let $x_{n}$ be a sequence of points of $B^{3} \backslash\{0\}$ tending to $x_{0}$. Then since $I$ is continuous, we can find a sequence of points $y_{n}$ lying on the leaves through $x_{n}$ which approach $y$. Then

$$
I(y)=\lim I\left(y_{n}\right)=\lim I\left(x_{n}\right)=I\left(x_{0}\right),
$$

showing $\gamma_{2}$ is a leaf with orientation induced from the oriented maximal geodesic $\gamma_{1} \cup\{0\} \cup \gamma_{2}$ of $B^{3}$.

LEMMA 4.6. In Case 2, the foliation extends uniquely to a $C^{0}$ foliation on $B^{3}$. This foliation is $C^{\omega}$.

Proof. By Lemma 4.5 the leaves $\gamma_{1}, \gamma_{2}$ of $F$ can be joined together to give a geodesic $\gamma$ through 0 . We claim that in this way we obtain a $C^{0}$ foliation $\widetilde{F}$ with leaves those of $\mathscr{F}$ but with $\gamma_{1}$ and $\gamma_{2}$ replaced by $\gamma$. To show this is a foliation we must construct a foliated chart on a neighbourhood of 0 . To do this consider the solid cylinder $\mathscr{E}=U \times(0, r) \subset \mathbb{R}^{3}$, which we obtain by identifying the slice $W$ with an open neighbourhood $U$ of 0 in $\mathbb{R}^{2}$. Let $d$ be the distance from $x_{0}$ to 0 along $\gamma_{1}$ and choose $r=d+\varepsilon$ for some $\varepsilon>0$. Assume that $W$ and $\varepsilon$ are chosen sufficiently small that for $x \in W$, the geodesic leaf through $x, \gamma_{x}(t)$ is contained in $B^{3}$ for $t \in(0, r)$. Orient $\gamma_{x_{0}}$ to point towards the center 0 of the ball and orient nearby $\gamma_{x}$ accordingly.

Define $f: \mathscr{C} \rightarrow B^{3}$ by $f(y, t)=$ the point obtained by moving a distance $t$ along the geodesic leaf passing through $y \in U \approx W$. Put $f(\mathscr{C})=V \subset B$. Then $f$ is injective since no two geodesics intersect. Furthermore $f$ is continuous. By invariance of domain [10, Theorem 18.9], $f$ has a continuous inverse $f^{-1}: V \rightarrow \mathscr{C}$ and so $f$ is a homeomorphism onto $V$. By construction $V$ contains 0 . Thus $f^{-1}$ is the desired foliated chart on a neighbourhood of 0 and so $\mathscr{F}$ Is a $C^{0}$ foliation on $B^{3}$. That this is a $C^{\omega}$ foliation follows from Theorem 2.19.

Proof of Theorem 4.1. Simply combine Lemmas 4.3, 4.4, 4.5 and 4.6.

Proof of Theorem 4.2. Let $\mathscr{F}$ be the foliation associated to $\phi$, that is, with leaves the connected components of the fibres of $\phi$. Then by Theorem 4.1, either (1) the foliation is the radial foliation, or (2) the foliation extends to a $C^{\infty}$ foliation $\widetilde{F}$ on $B^{3}$.

In Case (1), $\phi$ is constant on radii and therefore factors into radial projection $\pi: B^{3} \backslash\{0\} \rightarrow S^{2}, x \mapsto x /|x|$ followed by a map $\zeta: S^{2} \rightarrow N$. Now 
the $S^{2}$ can be identified with a geodesic sphere in $B^{3}$ centre 0 and $\zeta$ may be identified with $\phi$ restricted to this sphere. Therefore $\zeta$ is smooth. Since the geodesic sphere is everywhere horizontal, horizontal weak conformality of $\phi$ implies weak conformality of $\zeta$.

In Case (2), with the notations of Lemmas 4.5 and 4.6, first note that if $x \in \gamma_{1}, y \in \gamma_{2}$ since $\phi$ is continuous,

$$
\phi(y)=\lim \phi\left(y_{n}\right)=\lim \phi\left(x_{n}\right)=\phi(x) .
$$

Set $\phi(0)=\phi\left(\gamma_{1}\right)=\phi\left(\gamma_{2}\right)$. Then the foliation associated to $\phi$ is precisely the extended foliation $\mathscr{F}$. Since this is $C^{\infty}$ so is $\phi$.

(B) Global theory. Let $U$ be a connected open subset of a three-dimensional Riemannian manifold. Say that $\mathscr{F}$ (respectively $\phi$ ) is a conformal foliation by geodesics (a harmonic morphism) on $U$ with an isolated singularity at $x_{0} \in U$, if it is defined and $C^{\infty}$ on $D \backslash\left\{x_{0}\right\}$ where $D$ is a disc centre $x_{0}$, but does not have a $C^{\infty}$ extension to $D$.

REMARK. If $\mathscr{F}$ (respectively $\phi$ ) has other singularities, simply replace $U$ by $U \backslash \mathscr{E}$, where $\mathscr{E}$ is the set of other singularities. Thus theorems below apply to foliations and harmonic morphisms with any number and type of singularities provided one of them is an isolated singularity.

THEOREM 4.7. Let $\mathscr{F}$ be a conformal foliation by geodesics with an isolated singularity of a connected open subset $U$ of $\mathbb{E}^{3}=\mathbb{R}^{3}, S^{3}$ or $\mathbb{H}^{3}$. Then, up to isometry of $\mathbb{E}^{3}, F$ is the restriction of one of the foliations of Example 2.9, that is, radial foliation of $\mathbb{R}^{3}$ or $\mathbb{H}^{3}$, or foliation by half great circles through the poles of $S^{3}$.

Proof. By Theorem 4.1, $\mathscr{F}$ must be the radial foliation on some geodesic ball $B^{3}$ of $U$ centred on the singularity. Performing an isometry of $\mathbb{E}^{3}$, we can assume this singularity is at 0 (for $\mathbb{R}^{3}, \mathbb{H}^{3}$ ) or at $(1,0,0,0$ ) (for $S^{3}$ ). But radial foliation on $B^{3}$ is the restriction of one of the global radial foliations $\mathscr{F}_{0}$ of Examples 2.9. Since any conformal foliation is real analytic, $\mathscr{F}$ and $\mathscr{F}_{0}$ must agree on their common domain, that is, $\mathscr{F}$ is the restriction of $\mathscr{F}_{0}$.

THEOREM 4.8. Let $\phi$ be a harmonic morphism with an isolated singularity from a connected open subset $U$ of $\mathbb{E}^{3}=\mathbb{R}^{3}, S^{3}$ or $\mathbb{H}^{3}$ to a two-dimensional Riemannian manifold $N$. Then, up to isometry of $\mathbb{E}^{3}, \phi$ is the restriction of one of the standard radial projections (Examples 1.3), followed by a weakly conformal map from $S^{2}$ to $N$. (Hence $N$ is conformally equivalent to $S^{2}$ or $\mathbb{R} P^{2}$.) 
Proof. By Theorem 4.2, $\phi$ restricted to some ball $B^{3}$ of $U$ is radial projection to the centre of the ball followed by a weakly conformal map $\zeta: S^{2} \rightarrow N$. But the radial projection is the restriction of one of the harmonic morphisms $\phi_{0}$ of Examples 1.3. Since $\phi$ and $\zeta \circ \phi_{0}$ agree on $B^{3}$, by real analyticity they must agree on their common domain, i.e. $\phi$ is the restriction of $\phi_{0}$ followed by the weakly conformal map $\zeta: S^{2} \rightarrow N$.

\section{Harmonic morphisms and conformal foliations by geodesics of $R P^{3}$}

As explained in the introduction, our results for simply connected space forms imply results for other space forms. As an example we give a complete treatment for $\mathbb{R} P^{3}=S^{3} / \mathbb{Z}_{2}$.

EXAMPLES 5.1. (i) The Hopf map $S^{3} \rightarrow S^{2}$ factors to a map $\mathbb{R} P^{3} \rightarrow S^{2}$ which we still call the Hopf map.

(ii) The radial projection $S^{3} \backslash\{(1,0,0,0),(-1,0,0,0)\} \rightarrow S^{2}$ down geodesics through the poles factors to a map $\mathbb{R} P^{3} \backslash\{[1,0,0,0]\} \rightarrow \mathbb{R} P^{2}$, given by $\left[x_{1}, x_{2}, x_{3}, x_{4}\right] \mapsto\left[x_{2}, x_{3}, x_{4}\right]$. $\mathbb{R} P^{3}$.

(iii) Associated to the Hopf map is a conformal foliation by geodesics of

(iv) Associated to the projection $\mathbb{R} P^{3} \backslash\{[1,0,0,0]\} \rightarrow \mathbb{R} P^{2}$ is a conformal foliation by geodesics with an isolated singularity of $\mathbb{R} P^{3}$.

THEOREM 5.2. Let $\mathscr{F}$ be a conformal foliation by geodesics of $\mathbb{R} P^{3}$. Then, up to isometry, $\mathscr{F}$ is the foliation associated to the Hopf map $\mathbb{R} P^{3} \rightarrow S^{2}$ (Example 5.1(iii)).

THEOREM 5.3. Let $\phi: \mathbb{R} P^{3} \rightarrow N$ be a nonconstant harmonic morphism to a two-dimensional Riemannian manifold. Then up to isometry, $\phi$ is the Hopf map $\mathbb{R} P^{3} \rightarrow S^{2}$ (Example 5.1(i)) followed by a weakly conformal map $S^{2} \rightarrow N$. (In particular $N$ is conformally equivalent to $S^{2}$ or $\mathbb{R} P^{2}$.)

Proof of Theorem 5.2. $F$ lifts to a conformal foliation by geodesics of $S^{3}$. By Theorem 2.14, up to isometry, this must be the foliation corresponding to the Hopf map $S^{3} \rightarrow S^{2}$. The theorem follows.

Proof of TheOREM 5.3. Let $\pi: S^{3} \rightarrow \mathbb{R} P^{3}$ be the standard projection. Then by [4, Theorem 5.1], $\phi \circ \pi$ must be the Hopf map $\phi_{0}: S^{3} \rightarrow S^{2}$ 
followed by a weakly conformal map $\zeta: S^{2} \rightarrow N^{2}$.

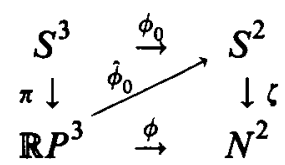

Let $\hat{\phi}_{0}: \mathbb{R} P^{3} \rightarrow S^{2}$ be the Hopf map of Example 5.1(i). Then $\phi \circ \pi=$ $\zeta \circ \phi_{0}=\zeta \circ \hat{\phi}_{0} \circ \pi$. Therefore, since $\pi$ is surjective, $\phi=\zeta \circ \hat{\phi}_{0}$ and the theorem is proved.

Regarding harmonic morphisms and conformal foliations by geodesics with isolated singularities of $\mathbb{R} P^{3}$, we have

THEOREM 5.4. Let $F$ be a conformal foliation by geodesics with an isolated singularity of a connected open subset $U$ of $\mathbb{R} P^{3}$. Then, up to isometry of $\mathbb{R} P^{3}, \mathscr{F}$ is the restriction to $U$ of the foliation associated to the projection $\mathbb{R} P^{3} \backslash\{[1,0,0,0]\} \rightarrow \mathbb{R} P^{2}$ (Example 5.1(iv)).

THEOREM 5.5. Let $\phi$ be a harmonic morphism to a two-dimensional Riemannian manifold $N$ with an isolated singularity defined on $\mathbb{R} P^{3}$ or more generally on an open subset $U$ of $\mathbb{R} P^{3}$ such that $\pi^{-1}(U) \subset S^{3}$ is connected, where $\pi: S^{3} \rightarrow \mathbb{R} P^{3}$ is the standard projection. Then, up to isometry of $\mathbb{R} P^{3}$, $\phi$ is the restriction to $U$ of projection $\mathbb{R} P^{3} \backslash\{[1,0,0,0]\} \rightarrow \mathbb{R} P^{2}$ (Example 5.1(iii)) followed by a weakly conformal map $\mathbb{R} P^{2} \rightarrow N$.

Proof of Theorem 5.4. By an isometry of $\mathbb{R} P^{3}$ we may assume that the isolated singularity is at $[1,0,0,0]$. By Theorem 4.1 , in the neighbourhood of the singularity, $\mathscr{F}$ must be the radial foliation to $[1,0,0,0]$. But this foliation is the restriction of that of Example 5.1(iv). By real analyticity (Remark 2.12(ii)), they must agree on their common domain.

Proof OF TheOREM 5.5.

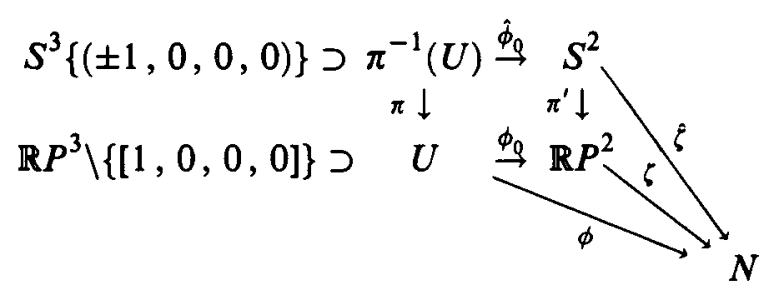

Let $\pi^{\prime}: S^{2} \rightarrow \mathbb{R} P^{2}$ be the standard projection. By Theorem 4.8 , since $\pi^{-1}(U)$ is connected, up to isometry of $S^{3}, \phi \circ \pi$ is the restriction of the 
standard radial projection $\hat{\phi}_{0}: S^{3} \backslash\{( \pm 1,0,0,0)\} \rightarrow S^{2}$ (Example 1.3(ii)) followed by a weakly conformal map $\hat{\zeta}: S^{2} \rightarrow N$. Now for any $y \in S^{2}$, there exists an $x \in \pi^{-1}(U)$ such that $\hat{\phi}_{0}(x)=y$ since $\hat{\phi}_{0}$ is surjective (on any neighbourhood of $(1,0,0,0)$ (or $(-1,0,0,0))$, and so

$$
\hat{\zeta}(-y)=\hat{\zeta}\left(\hat{\phi}_{0}(-x)\right)=\phi(\pi(-x))=\phi(\pi(x))=\hat{\zeta}\left(\hat{\phi}_{0}(x)\right)=\hat{\zeta}(y),
$$

therefore $\hat{\zeta}$ factors to a weakly conformal map $\zeta: \mathbb{R} P^{2} \rightarrow N$. But then $\phi \circ \pi=\zeta \circ \pi^{\prime} \circ \hat{\phi}_{0}=\zeta \circ \phi_{0} \circ \pi$ where $\phi_{0}: \mathbb{R} P^{3} \backslash\{[1,0,0,0]\} \rightarrow \mathbb{R} P^{2}$ is the standard projection (Example 5.1(ii)) and since $\pi$ is surjective this implies $\phi=\zeta \circ \phi_{0}$ and the result is proven.

REMARK. The result is false if $\pi^{-1}(U)$ is not connected. For example, if $U$ is a geodesic disc of $\mathbb{R} P^{3}$ centre $[1,0,0,0]$, radial projection $U \backslash\{[1,0,0,0]\} \rightarrow S^{2}$ does not factor through $\mathbb{R} P^{2}$.

\section{Acknowledgements}

The authors would like to thank I. Aitchison, H. Rubinstein and N. Trudiger for helpful comments on this work. The first named author would also like to thank the Science and Engineering Research Council for their financial support during the preparation of this paper.

\section{References}

[1] P. Baird, 'Harmonic morphisms onto Riemann surfaces and generalized analytic functions', Ann. Inst. Fourier (Grenoble) 37 (1987), 135-173.

[2] P. Baird, 'Harmonic morphisms and circle actions on 3- and 4-manifolds', Ann. Inst. Fourier (Grenoble) 40 (1990), 177-212.

[3] P. Baird and J. Eells, A conservation law for harmonic maps, in: E. Looijenga, D. Siersma, F. Takens (eds.), Geometry Symposium Utrecht 1980, Proceedings, Lecture Notes in Mathematics, Vol. 894, pp. 1-25, Springer, Berlin, Heidelberg, New York, 1981.

[4] P. Baird and J. C. Wood, 'Bernstein theorems for harmonic morphisms from $R^{3}$ and $S^{3}$, Math. Ann. 280 (1988), 579-603.

[5] A. Bernard, E. A. Campbell, and A. M. Davie, 'Brownian motion and generalized analytic and inner functions', Ann. Inst. Fourier (Grenoble) 29 (1979), 207-228.

[6] J. B. Conway, Functions of one complex variable, Springer, Berlin, Heidelberg, New York, 1983.

[7] J. Eells and J. C. Polking, 'Removable singularities of harmonic maps', Indiana Univ. Math. J. 33 (1984), pp. 859-871.

[8] J. Eells and J. H. Sampson, 'Harmonic mappings of Riemannian manifolds, 'Amer. J. Math. 86 (1964), 109-160. 
[9] B. Fuglede, 'Harmonic morphisms between Riemannian manifolds', Ann. Inst. Fourier (Grenoble) 28 (1978), 107-144.

[10] M. Greenberg, Lectures on algebraic topology, Benjamin, New York, Amsterdam, 1966.

[11] R. E. Greene and H. Wu, 'Embeddings of open Riemannian manifolds by harmonic functions', Ann. Inst. Fourier (Grenoble) 12 (1962), 415-471.

[12] G. Hector and U. Hirsch, Introduction to the geometry of foliations, parts $A$ and $B$, Aspects of Mathematics, Vieweg, Brauschweig, Wiesbaden, 1981.

[13] M. Heins, Complex function theory, Academic Press, 1968.

[14] L. L. Helms, Introduction to potential theory, Wiley, New York, London, Sydney, Toronto, 1969.

[15] N. Hitchin, 'Monopoles and geodesics', Comm. Math. Phys. 83 (1982), 579-602.

[16] T. Ishihara, 'A mapping of Riemannian manifolds which preserves harmonic functions', J. Math. Kyoto University 19 (1979), 215-229.

[17] A. Morgan, 'Holonomy and metric properties of foliations in higher codimensions', Proc. Amer. Math. Soc. 58 (1976), 255-261.

[18] B. L. Rienhart, Differential geometry of foliations, Ergebnisse Math., Vol. 99, SpringerVerlag, Berlin, Heidelberg, New York, 1983.

[19] N. Steenrod, The topology of fibre bundles, Princeton University Press, 1951.

[20] I. Vaisman, 'Conformal foliations', Kodai Math. J. 2 (1979), 26-37.

[21] J. Wolf, Spaces of constant curvature, McGraw-Hill, New York, St. Louis, San Francisco, Toronto, London, Sydney, 1967.

[22] J. C. Wood, Harmonic morphisms foliations and Gauss maps, in: Y. T. Siu (ed.), Complex differential geometry and nonlinear differential equations, Contemporary Mathematics, vol. 49, pp. 145-183, Amer. Math. Soc., Providence, R.I., 1986.

\section{Department of Pure Mathematics \\ University of Leeds \\ Leeds LS29JT \\ United Kingdom}

